\title{
Proof-checking Euclid
}

\section{Michael Beeson ${ }^{1}$ (D) . Julien Narboux ${ }^{2} \cdot$ Freek Wiedijk $^{3}$}

Published online: 10 January 2019

(C) The Author(s) 2019

\begin{abstract}
We used computer proof-checking methods to verify the correctness of our proofs of the propositions in Euclid Book I. We used axioms as close as possible to those of Euclid, in a language closely related to that used in Tarski's formal geometry. We used proofs as close as possible to those given by Euclid, but filling Euclid's gaps and correcting errors. Euclid Book I has 48 propositions; we proved 235 theorems. The extras were partly "Book Zero", preliminaries of a very fundamental nature, partly propositions that Euclid omitted but were used implicitly, partly advanced theorems that we found necessary to fill Euclid's gaps, and partly just variants of Euclid's propositions. We wrote these proofs in a simple fragment of first-order logic corresponding to Euclid's logic, debugged them using a custom software tool, and then checked them in the well-known and trusted proof checkers HOL Light and Coq.
\end{abstract}

Keywords Euclid · Proof-checking $\cdot$ Euclidean geometry $\cdot$ HOL light $\cdot$ Coq

Mathematics Subject Classification (2010) 03B30 · 51M05

\section{Introduction}

Euclid was the "gold standard" of rigor for millenia. The Elements of Euclid set the standard of proof used by Isaac Newton in his Principia and even Abraham Lincoln claimed to

Freek Wiedijk

freek@cs.ru.nl

Julien Narboux

narboux@unistra.fr

Michael Beeson

beesonpublic@gmail.com; profbeeson@gmail.com

1 Department of Mathematics, San Jose State University, 185 View Ct, Aptos, CA 95003, USA

2 ICube, UMR 7357 CNRS, University of Strasbourg, PôleAPI Bd Sébastien Brant, BP10413, 67412 Illkirch, France

3 Institute for Computing and Information Sciences, Radboud University, Toernooiveld 212, 6525 EC Nijmegen, The Netherlands 
have read all ten books of Euclid and learned from it how to prove something in court. The Elements also inspired the form of the American Declaration of Independence. In the modern era, beginning already in the nineteenth century, the standards of proof in mathematics became more demanding, and the imprecisions and gaps in Euclid were more apparent than before. Even before that time, some mathematicians focused on the perceived flaw that the Fifth Postulate (the "parallel postulate", or "Euclid 5") was less intuitively evident than the other four. ${ }^{1}$ Efforts to remove this "flaw" by proving Euclid 5 led to the development of non-Euclidean geometry, showing that in fact Euclid 5 was a necessary postulate, not a flaw.

Nevertheless there are flaws in Euclid, and we want to discuss their nature by way of introduction to the subject. ${ }^{2}$ The first gap occurs in the first proposition, I.1, in which Euclid proves the existence of an equilateral triangle with a given side, by constructing the third vertex as the intersection point of two circles. But why do those two circles intersect? Euclid cites neither an axiom nor a postulate nor a common notion. This gap is filled by adding the "circle-circle" axiom, according to which if circle $C$ has a point inside circle $K$, and also a point outside circle $K$, then there is a point lying on both $C$ and $K$.

There is, however, a second gap in the proof of I.1. Namely, the proposition claims the existence of a triangle, which by definition is (or at least is determined by) three noncollinear points. ${ }^{3}$ Why are the three points in question not all on a line? Intuitively, if they were, then one would be between the other two, violating Euclid's common notion that the part is not equal to the whole, since all the sides are equal.

A formal proof of Prop. I.1 cannot follow Euclid in ignoring this issue. The proposition that if $B$ is between $A$ and $C$ then $A B$ is not equal to $A C$ therefore must precede proposition I.1, unless one is to consider it as an axiom formalizing one of Euclid's common notions. In the next section, we discuss the axioms and postulates of Euclid, and how we have chosen to render them in modern first-order form.

The two gaps in I.1 illustrate two common failures. Many of the gaps in Euclid fall into one of these categories:

(i) A failure to prove that a point clearly shown in the diagram actually exists, e.g. that two lines really do intersect, or as in I.1 two circles.

(ii) A failure to prove that points shown in the diagram to be non-collinear, are in fact non-collinear.

(iii) A failure to prove that a point shown in the diagram to be between two other points, is in fact between those points.

Another example of an error of type (i) is in the second paragraph of Prop. I.44, "let $F G$ be drawn through to $H$ ". Here $H$ has not been proved to exist, a strange omission in that a few lines later Euclid does feel the need to use Postulate 5 to prove that $K$ exists; but then two lines later "let $H A, G B$ be produced to the points $L, M$ ". That is, the lines shown as intersecting at $L$ and $M$ do in fact intersect-but Euclid offers no justification for that line of the proof. There are dozens of such fillable gaps in Euclid's proofs, some more easily filled than others.

\footnotetext{
${ }^{1}$ Proclus [38], writing in the fifth century, said that Euclid 5 needed a proof, and tried to supply one; and he was not the first, since he also criticized a previous attempt by Ptolemy. See [21], Ch. 5 for further history.

${ }^{2}$ It is customary to refer to the propositions of Euclid with notation like I.44, which means Proposition 44 of Book I."

${ }^{3}$ Euclid never defines triangle, although he does define right triangle and equilateral triangle. Instead he mentions trilateral figure, which is "contained by three straight lines." We read that to imply non-collinearity, for otherwise there would be only one line, and nothing "contained."
} 


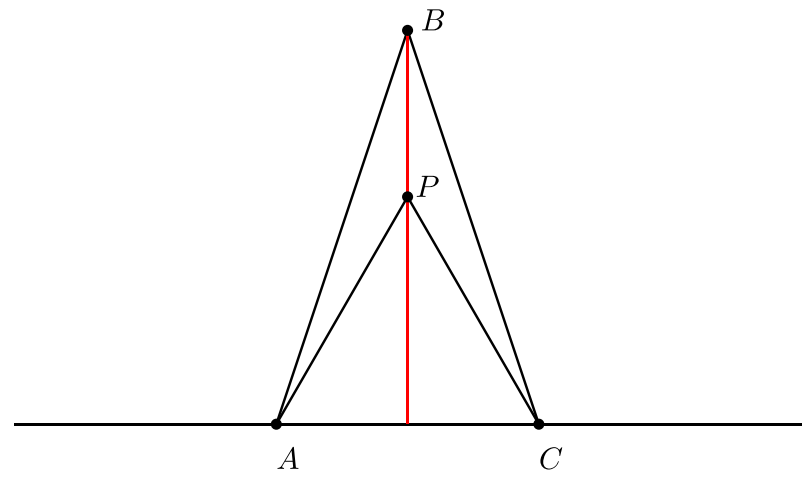

Fig. 1 Euclid's proof of I.9. What if $B=P$ ? Even if $B \neq P$, why does $B P$ lie in the interior of $A B C$, i.e., why does it meet $A C$ ?

Not every error in Euclid falls into these categories, however. Consider Prop. I.9, in which Euclid bisects an angle. The method is to lay off the same length on both sides of the angle, and then construct an equilateral triangle on the base thus formed. Connecting the vertex of the original angle with the vertex of the equilateral triangle, we get the angle bisector (Fig. 1).

Oops, but the two points might coincide! Well, in that case we ought to have drawn the other equilateral triangle, on the other side of the base. But Prop. I.1 did not provide for the construction of two triangles, and we cannot easily construct "the other one." We certainly need to expand the list of ways in which Euclid's proofs fall short by at least two more items:

(iv) A failure to prove that points shown in the diagram to be distinct are in fact distinct.

(v) A failure to show that points are on the same side (or opposite sides) of a line, as the diagram shows them to be.

Even if we could solve these problems, the proof of I.9 still would not be correct, since we would still need to show that the angle bisector constructed does in fact lie in the interior of the angle. That difficulty brings us to an important point. There is no "dimension axiom" in Euclid to guarantee that we are doing plane geometry. Hence "circles" are really "spheres", and rather than just two equilateral triangles on a given base, there are infinitely many. $B P$ might not even lie in the same plane as $A C$ ! So even if the vertex of the equilateral triangle is distinct from the vertex of the original angle, why does the line between them lie in the interior of the angle? In fact that would be a problem even in two dimensions-an example of (iii) above.

Even if it were possible to fix that problem by adding a dimension axiom, that would not be desirable. Euclid didn't just forget to write down a dimension axiom. In Book X and beyond, Euclid works in three-space, and the culmination of the whole series of books is the study of the Platonic solids. Hence it is clear that Euclid is not meant to be restricted to plane geometry. In the absence of a dimension axiom, it is good advice to the reader to visualize "circles" as spheres. Then the two circles used in I.1 have not just two but many intersection points. The circle-circle axiom only guarantees the existence of one intersection point. ${ }^{4}$

\footnotetext{
${ }^{4}$ Since Euclid did not state the axiom, one could consider strengthening it to state the existence of an intersection point on a given side of the line connecting the centers of the circles. But Euclid also did not say in I.1 or I.9 anything about this problem; and we are able to prove I.9 from the more fundamental form of circle-circle.
} 
Therefore we conclude that Euclid's proof of I.9 is fatally flawed. We prove it another way: by first showing how to bisect a line, and then using that to bisect an angle. The simple and ingenious proof that a line can be bisected if it is the base of an isosceles triangle was apparently not noticed until Gupta's thesis in 1965 [23], but could very well have been discovered by Euclid.

The fact that there is no dimension axiom is not always remembered in Book I, and is the source of several difficulties. A good example is Prop. I.7, which says that if $A B C$ is a triangle and $A B D$ is another triangle congruent to $A B C$ (that is, $A C=A D$ and $B C=$ $B D$ ), and $C$ and $D$ are on the same side of $A B$, then $C$ is equal to $D$. Look at the figure for I.7; in two dimensions it appears contradictory, but as soon as you think that it might be in three dimensions, the contradiction disappears. The statement of Prop. 7 does correctly include the hypothesis that $C$ and $D$ are on the same side of $A B$, but Euclid never uses that hypothesis in the proof. That is not surprising, since he never defined "same side", so he had no possible way to use that hypothesis. It should have been used to verify the claim that angle $D C B$ is less than angle $D C A$, because there is a point of intersection of $A D$ and $C B$. This turns out to not be provable, even after proving a number of more basic propositions in "Book Zero"; we could not prove I.7 without using "dropped perpendiculars", which are only constructed much later in Euclid.

A well-known geometer told us "there are no errors in Euclid", in the sense that the statements of all the propositions are true in the plane. If we supply Tarski's definition of "same side", an even stronger version of that claim is true: the statements of all the propositions are true in every finite-dimensional space $\mathbb{R}^{n}$. However, the same cannot be said of the proofs. Many of these have problems like those of I.9 and I.7; that is, we could fix these problems only by proving some other propositions first, and the propositions of the first half of Book I had to be proved in a different order, namely 1,3,15,5,4,10,12,7,6,8,9,11, and in some cases the proofs are much more difficult than Euclid thought. After proving those early propositions, we could follow Euclid's order better, and things went well until Prop. 44. In Propositions 44 and 47 there are numerous points of difficulty, which took extra propositions to resolve. As one example we mention the proposition that every square is a parallelogram, which Euclid uses implicitly in proving Prop. 47. (By definition a square has equal sides and four right angles, and a parallelogram is a quadrilateral with opposite sides parallel.) Euclid could and should have proved that.

The aims of the project reported on in this paper are as follows:

(i) Fix Euclid's axioms (and common notions), using an axiom system rather close to Euclid's, but including axioms about betweenness that Euclid omitted, and with other changes discussed below.

(ii) Give correct proofs of all the propositions in Book I from the new axioms, following Euclid's proofs as closely as possible.

(iii) Show that those proofs are indeed correct by checking the proofs using the proofchecking programs HOL Light and Coq.

(iv) Show that the axioms are indeed correct by computer-checking proofs that the axioms hold in the Cartesian plane $\mathbb{R}^{2} .5$

Accordingly, in this paper we limit the discussion of geometry to the description of the axioms, the description of a few specific flaws in Euclid's reasoning, and a discussion of

\footnotetext{
${ }_{5}^{5}$ The axioms hold in $\mathbb{F}^{n}$, where $\mathbb{F}$ is any Euclidean field (an ordered field in which positive elements have square roots), but we have not computer-checked a proof of that fact, even for $n=2$; we did check $\mathbb{R}^{2}$.
} 
Euclid's notion of "equal figures" that is necessary to verify the axioms we use about that notion. Our focus in this paper is on proof checking. What we report on here would still have been worth doing, even if there were no gaps or errors in Euclid. The details of our proofs, and a discussion of the errors in the original proofs of Euclid, will be published separately, with a focus on the geometry and on the correspondence between the axioms and proofs of Euclid and those of our formal development. That will necessitate a longer discussion than is possible here.

The formal proofs themselves, as well as the PHP and ML scripts that we used, are available on the Web. Look for links to them at http://www.michaelbeeson.com/research/ CheckEuclid/index.php. They are also available as ancillary files to the version of this paper posted on ArXiv.

\section{History}

A scholarly history of the previous attempts to axiomatize Euclidean geometry would require a long paper in itself; we offer only a few highlights here. But before beginning, we point out that axiomatizing Euclidean geometry is not quite the same as axiomatizing Euclid: the former aims at an axiom system that permits the derivation of important theorems, regardless of whether the axioms or the proofs are similar to Euclid's axioms and proofs; the latter pays attention to those points.

The best known attempt is Hilbert's 1899 book [25]. Hilbert had been a vocal proponent of the axiomatic method, and his book was probably meant partly to illustrate that method on the example of geometry. First-order logic was in its infancy and Hilbert's system was not first order. He made use of Archimedes's axiom, and his continuity "axiom" is a strange mixture of logic and model theory. ${ }^{6}$ The fundamental idea to use betweenness and congruence as primitive relations goes back to Pasch[35]. Further contributions by Mollerup [32], Veronese [44], Pasch [35], and Peano[36] are discussed below in connection with the axioms they helped to develop. After Hilbert, the most important work is the axiom system of Tarski. This is a first-order system, and not only is it first-order, it is points-only, meaning that there are variables only for points. Lines are given by two points, and angles by three points, and equality between angles is a defined notion. This fits Euclid very well: Euclid almost always refers to lines by two points, and angles by three points. Tarski's system was developed in 1927, but publication was long delayed; for the history see the introduction to (the Ishi press edition of) [40]. Although a development of Euclid in Tarski's system could have been done in the 1960s at Berkeley, it was not done. Instead efforts focused on reducing the number of Tarski's axioms by finding dependencies, and on proving fundamental results like the existence of perpendiculars and midpoints without using the parallel postulate or any continuity axioms. The results of these efforts finally appeared in [40], which contains the remarkable results of [23].

In spite of a century of effort, in 2017 we did not possess any formal analysis of Euclid's proofs, for Hilbert and Tarski had both aimed at avoiding the circle axioms and developing segment arithmetic, while Euclid uses the circle axioms and the last half of Book I is based on the equal-figure axioms (discussed in Section 7). Even if we add the circle axioms, the last half of Book I can be proved in Hilbert or Tarski's theories only after the long and difficult development of segment arithmetic, so that "equal figures" can be defined as "equal area."

\footnotetext{
${ }^{6}$ It can be viewed as a second-order axiom involving quantification over models of the other axioms.
} 
It is now 35 years since the publication of [40], and meantime, the technology of proof checking by computer has advanced. Our predecessors stopped at the threshold, so to speak, by working on neutral geometry and minimal axioms systems, instead of formalizing Euclid. We serendipitously find ourselves in the situation where it is possible for us to take up that task, and also to verify (using existing computer proof checkers) that our proofs are flawlessly correct.

\section{Language}

Euclid did understand the fundamental point that not every fact can be proved; the first fact accepted could not be proved because there would be nothing to prove it from. But he did not understand that similarly, not every concept can be defined. Thus he famously attempted to define "point" and "line" and "rectilinear angle." (The Greeks also considered "angles" formed by curved sides.) These natural-language "definitions" are not mathematical usable; so in practice Euclid treated points, angles, circles, and lines all as primitive notions. In addition, Euclid treated "figures" as a primitive notion, in the sense that he never made use of the circular and vague definitions he offered. ${ }^{7}$ In Book I, only triangle and quadrilaterals are used. Euclid also accepted concepts of "equal" and "greater than" for each of points, angles, circles, lines, and figures without definition (but curiously, there is no "greater than" for figures). In the middle of the nineteenth century, it was recognized that "betweenness" and "equidistance" were good primitives for geometry, and later it was realized that it is possible to work with angles represented by triples of points, instead of taking them as primitive, so angle equality and inequality are defined concepts. That is what we do in our formal work. Thus all our axioms, except those mentioning circles, are formulated in a "points-only" language, in which the fundamental relations are betweenness and equidistance.

Betweenness is a 3-ary relation $\mathbf{B}(A, B, C)$, which Euclid wrote as " $B$ lies on the finite straight line $A C$ ", or (for example in Prop. I.14) as " $A B$ and $B C$ lie in a straight line." We interpret $\mathbf{B}$ as strict betweenness, i.e. the endpoints do not lie on the line. ${ }^{8}$

Collinearity is the relation $L(A, B, C)$ expressing that either two of the points are equal or one lies between the other two. This is a statement about points only. It seems that for Euclid, lines were primitive objects, rather than sets, and the incidence relation (point lies on line) too fundamental even to notice, as it does not occur in the list where "point" and "line" are "defined." Whether close to Euclid or not, we use the first-order formulations of betweenness (a primitive) and collinearity (defined). ${ }^{9}$

"Equidistance" is a 4-ary relation representing the congruence of finite lines, " $A B$ is equal to $C D$ ". Euclid, or at least his translator Heath, used "equal" rather than "congruent". (The word "equidistance" is also not faithful to Euclid, who never spoke of distances.)

There is one exception to our "points-only" approach. In order to follow Euclid more closely, we allow giving a name to a circle. Circles are given by point and radius, so we can say " $J$ is a circle with center $P$ and radius $A B$." (Here the "radius" is not a number but

\footnotetext{
${ }^{7}$ A figure is that which is contained by a boundary; a boundary is that which is an extremity of anything.

${ }^{8}$ Hilbert used strict betweenness; Tarski used non-strict betweenness, on purpose because the degenerate cases could be used to reduce the number of axioms. We use strict betweenness, on purpose to avoid degenerate cases that express unintended things and have to be separately worried about.

${ }^{9}$ Most of the time Euclid's lines are finite, which may confuse a modern reader at first, since today finite lines are called "segments", and "line" means "infinite line." Hilbert made (infinite) lines primitive objects, but treated finite lines (segments) as sets of points.
} 

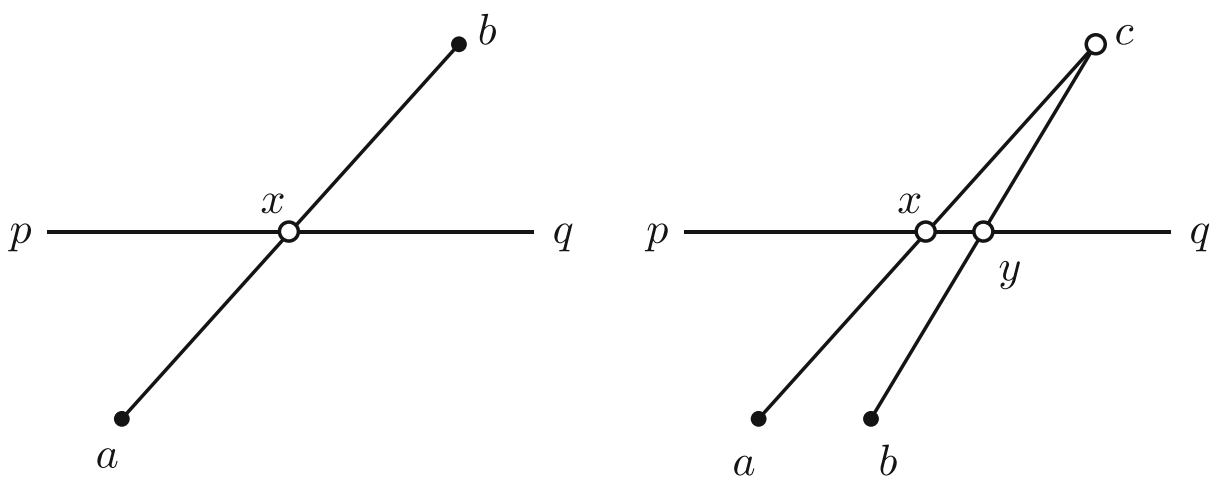

Fig. 2 (Left) $a$ and $b$ are on the opposite side of $p q$. (Right) $a$ and $b$ are on the same side of $p q$ if there exist points $x$ and $y$ collinear with $p q$, and a point $c$, such that $\mathbf{B}(a, x, c)$ and $\mathbf{B}(b, y, c)$

a finite line.) This is expressed by the formula $C I(X, C, A, B)$, using a primitive relation symbol $C I$. We do not make use of equality between variables of the sort for circles.

Euclid never gives circles a single-character name as we do. Nor does he name circles by center and radius, or center and point-on-circle. Instead he names circles by listing three points that lie on the circle. One of those points is a point that appears to exist in the diagram, and is conjured into existence without proof by the act of naming it. This naming technique papers over the lack of the circle-circle axiom in Euclid, and introduces a gap into the proof every time it is used. We therefore must deviate from Euclid's naming convention for circles.

Betweenness and equidistance are sufficient as primitive relations for elementary geometry, but the latter part of Euclid Book I uses another primitive relation, "equal figures", which is discussed in Section 7. We mention it here only to note that the complete definition of our language requires inclusion of the primitive relations discussed in Section 7.

\section{Definitions}

Euclid gives a long list of definitions at the beginning of Book I. We do the same. Euclid's list has some important omissions, notably "same side" and "opposite side". These are defined in Fig. 2. ${ }^{10}$ Euclid defined "square" but omitted "parallelogram" and "rectangle". He defined "parallel lines" to be lines that do not meet but lie in the same plane (thus illustrating that his omission of a dimension axiom was no accident!) On the other hand, he failed to define "lies in the same plane". Once we have defined "same side" as in Fig. 2, it is easy to define "lies in the same plane", as each line and point not on the line determine two half-planes, together making up a plane. In the formal statement of "same side", a line is specified by two distinct points $p, q$, and the incidence of $x$ on that line is expressed by " $p, q, x$ are collinear". Formally we use the relation $L(p, q, x)$ defined above in terms of betweenness. This definition exemplifies how one works with points only, avoiding the explicit mention of lines. The price one has to pay for this simplification is that one then has

\footnotetext{
${ }^{10}$ This definition is due to Tarski [40]. Hilbert had planes as a primitive concept, and discussed "same side" and "opposite side" only in the context of a fixed plane, using a definition that would not work without having planes as a primitive concept. Tarski's definition of "same side" is vital for making possible a points-only formalization that would work in more than two dimensions.
} 
to prove that it doesn't matter which particular points $p, q$ we chose to represent the line. That is, "same side" is invariant if $p$ and $q$ are changed to some other pair of distinct points each of which is collinear with $p$ and $q$.

Euclid's failure to define "lies in the same plane" leaves us to complete his definition of "parallel". First, we discuss the "not meeting" part of the definition. Lines in Euclid are finite, but "parallel" is about infinite lines. So " $A B$ does not meet $C D$ " means that no matter how those two finite lines are produced, the lengthened lines still do not have a point in common. In other words, there is no point collinear with both $A B$ and $C D$. On the other hand, " $A B$ crosses $C D$ " means that there is a point both between $A$ and $B$, and between $C$ and $D$.

We define "Tarski-parallel" by " $A B$ and $C D$ do not meet, and $C$ and $D$ lie on the same side of $A B$." This is clearly not what Euclid intended, as to Euclid it seems obvious that if $A B$ is parallel to $C D$ then $C D$ is parallel to $A B$. So we define instead that $A B$ is parallel to $C D$ if there is no point collinear with both $A B$ and $C D$, and there are four points $a, b, c, d$ with $a$ and $b$ collinear with $A B$, and $c, d$ collinear with $C D$, and $a d$ crosses $b c$. With this definition, there is a very short proof of the symmetry property. On the other hand, the two definitions can be proved equivalent. It follows that if $A B$ and $C D$ are parallel then $A$ and $B$ are on the same side of $C D$, which is quite often actually necessary, but never remarked by Euclid.

Euclid defines a square to be a quadrilateral with at least one right angle, in which all the sides are equal. ${ }^{11} \mathrm{He}$ does not specify that all four vertices lie in the same plane. This is not trivial to prove, but we did prove it, so Euclid's definition does not require modification. Euclid does not define "rectangle". One would like to define it as a quadrilateral with four right angles. It is a theorem that such a figure must lie in a plane. However, the proofs we found involve reasoning "in three dimensions". Even though Euclid Book I has no dimension axiom, and we must therefore be careful not to assume one, nevertheless all the proofs in Book I deal with planar configurations. We therefore define "rectangle" to be a quadrilateral with four right angles, whose diagonals cross, that is, meet in a point. This condition is one way of specifying that a rectangle lies in a plane. We can then prove that a rectangle is a parallelogram.

The Appendix contains a complete machine-generated list of our definitions.

\section{Angles}

We take only points and circles as primitive objects. Angles are treated as ordered triples of non-collinear points, $A B C$. The point $B$ is the vertex of the angle. Equality of angles is a 6-ary relation, which we write informally as "angle $A B C=a b c$ ". The definition is that there exist four points (one on each side of each angle) that form, with the vertices $B$ and $b$, two congruent triangles. (Two triangles are congruent, by definition, if all three pairs of corresponding sides are equal.) $)^{12}$ This definition does not permit "straight angles", "zero angles", or angles "greater than 180 degrees." Such "angles" are also not used in Euclid.

A point $F$ lies in the interior of angle $A B C$ if it is between two points lying on the two sides of the angle. Angle ordering is defined by $a b c<A B C$ if angle $a b c$ is equal to angle

\footnotetext{
${ }^{11}$ But in I.46 and I.47 the proofs work as if the definition required all four angles to be right, so we take that as the definition.

${ }^{12}$ This is not the same definition as used in [40], but it works, and seems simpler to us; perhaps the one in [40] seems simpler in the presence of function symbols for line extension.
} 
$A B F$, for some $F$ in the interior of $A B C$. Note that these definitions make sense without any dimension axiom; that is, they work fine in three-space.

We then have to prove as theorems those properties of angle equality and ordering that Euclid assumed as "common notions": reflexivity, symmetry, and transitivity of angle equality; the fact that angle $A B C$ is equal to angle $C B A$; transitivity of angle ordering. The fact that an angle cannot be both equal to and less than the same angle is quite difficult to prove, although taken for granted by Euclid in several proofs. That is, of course, the key result needed to prove antisymmetry and trichotomy for angle ordering.

Hilbert [25] took angles as primitive, and had an axiom about copying angles that specified the uniqueness of the copied angle. The uniqueness assumption builds in as an axiom the property that an angle cannot be both less than and equal to itself. Since this can be proved, it might be considered an imperfection to assume it as an axiom. While Hilbert took angles and equality of angles as primitive, he did define angle ordering just as we do. Because of the uniqueness part of his angle-copying axiom, he had no difficulty proving trichotomy.

\section{Axioms and postulates}

Euclid had three groups of what would now be called axioms: common notions, axioms, and postulates. The common notions were intended to be principles of reasoning that applied more generally than just to geometry. For example, what we would now call equality axioms. The axioms and postulates were about geometry. The distinction between an "axiom" and a "postulate", according to Proclus [38], p. 157, is that a postulate asserts that some point can be constructed, while an axiom does not. In modern terms an "axiom" is purely universal, while a postulate has an existential quantifier.

Heath's translation lists five common notions, five postulates, and zero axioms. Simson's translation [17] lists three postulates, twelve axioms, and zero common notions. The extra axioms are discussed by Heath on p. 223 of [18], where they are rejected.

\subsection{Euclid's common notions}

Euclid's first common notion is "things equal to the same thing are equal to each other." That is,

$$
a=c \wedge b=c \rightarrow a=b .
$$

Modern mathematicians would prefer

$$
a=c \wedge c=b \rightarrow a=b .
$$

But then, they need symmetry as a separate axiom $(a=b \rightarrow b=a)$, while that can be proved from Euclid's axiom above. We follow Euclid in this matter, although of course it is of no serious consequence. ${ }^{13}$

Euclid's fourth common notion is "Things which coincide with one another are equal to one another." We take this to justify reflexivity. We consider the transitivity, reflexivity, and symmetry of point equality to be part of logic.

\footnotetext{
${ }^{13}$ Apparently Euclid considered symmetry too obvious to mention. Or maybe, he considered " $A=B$ " and " $B=A$ " to just be different expressions of the same proposition, rather than different but equivalent propositions.
} 
When "equality" refers to congruence of lines, these principles correspond to three congruence axioms. We also need the axiom that $A B$ is equal to $B A$. In other words, Euclid's lines are not directional. Euclid never explicitly states this principle, but it is often necessary when formalizing his proofs. Perhaps Euclid would regard $A B$ and $B A$ as "coincident", in which case this axiom is covered by common notion 4 , quoted above.

Euclid himself used common notion 4 to justify his "proof" of the SAS principle I.3 by "superposition". We reject this proof, and that leaves only $A B=B A$ and reflexivity of congruence, point equality, and figures to correspond to common notion 4 .

Angle equality is a defined concept and its properties are theorems, not axioms. Equality of figures is axiomatized in Section 7.

Euclid's fifth common notion is "The whole is greater than the part." In our formalism, inequality of finite lines $A B<C D$ is defined as " $A B$ is equal to $C E$ for some $E$ between $C$ and $D$." Thus common notion 5 (for lines) is built into the definition. Then "the whole is not equal to the part" (for lines) becomes $A B<C D$ implies $A B$ is not equal to $C D$, which boils down to the principle partnotequal whole:

$$
\mathbf{B}(A, B, C) \rightarrow \neg A B=A C .
$$

This is a theorem, not an axiom, in our development.

Euclid's second common notion is "If equals be added to equals, the wholes are equal", and the third common notion is "If equals be subtracted from equals, the remainders are equal." Common notion 2 becomes our axiom sumofparts, which says that if $A B=a b$ and $B C=b c$ and $\mathbf{B}(A, B, C)$ and $\mathbf{B}(a, b, c)$, then $A C=a c$. Here $A B$ and $B C$ are "parts" and $A C$ is the "whole" made by "adding" the two lines. The related principle differenceofparts, corresponding to common notion 3, is proved, rather than assumed as an axiom; there is a fuller discussion in Section 6.6. Angle inequality is a defined notion and there are no axioms about it. These two common notions justify several of the axioms for figures, which are discussed in Section 7.

Equality also enjoys the substitution property for each predicate in our language:

$$
\begin{aligned}
\mathbf{B}(a, b, c) \wedge a & =A \wedge b=B \wedge c=C \rightarrow \mathbf{B}(A, B, C) \\
a b=c d \wedge a=A \wedge b & =B \wedge c=B \wedge d=D \rightarrow A B=C D
\end{aligned}
$$

and similarly for the predicates for "equal figures." In practice, the proofs are checked assuming the second-order property:

$$
a=b \rightarrow \forall P(P(a) \Longleftrightarrow P(b))
$$

which allows to avoid introducing a separate axiom for each predicate. That is, we do not actually use the substitution axioms for individual predicates, but allow the substitution of $A$ for $B$ in any derived formula, when $A=B$ or $B=A$ has been derived. Such substitutions are often needed in formalizing Euclid's proofs, so even if he did not explicitly state the principle, he understood it.

A complete machine-generated list of the common notions is in the Appendix.

\subsection{Betweenness axioms}

Euclid never made explicit mention of betweenness, ignored all places where it should have been proved, and had no axioms for proving betweenness statements. We will not discuss the historical origins of the following axioms, nor the possibilities for reducing their number (this is certainly not a minimal set, but the proofs required to eliminate some of these axioms 
are long and difficult.) We give them the names they are given in our formal development, which is why there are no spaces in those names.

$$
\begin{aligned}
& \overrightarrow{\mathbf{B}}(a, b, a) & & \text { betweennessidentity } \\
\mathbf{B}(a, b, c) & \rightarrow \mathbf{B}(c, b, a) & & \text { betweennessymmetry } \\
\mathbf{B}(a, b, d) \wedge \mathbf{B}(b, c, d) & \rightarrow \mathbf{B}(a, b, c) & & \text { innertransitivity }
\end{aligned}
$$

The following axiom is called connectivity and can be rendered in English as "If $B$ and $C$ lie on the finite straight line $A D$, and neither is between $A$ and the other, then they are equal."

Formally:

$$
\mathbf{B}(a, b, d) \wedge \mathbf{B}(a, c, d) \wedge \neg \mathbf{B}(a, b, c) \wedge \neg \mathbf{B}(a, c, b) \rightarrow b=c
$$

This principle was expressed in antiquity as "a straight line cannot enclose an area."14 It is closely related to the principle known in modern times as "outer connectivity", which says that if line $A B$ has two extensions $C$ and $D$ then either $C$ is between $B$ and $D$ or $D$ is between $B$ and $C$. We prove outer connectivity as a theorem from the connectivity axiom. ${ }^{15}$

\subsection{Extension of lines}

Euclid postulated that every line can be extended, but (at least in Heath's translation) did not say by how much. ${ }^{16}$ We render Euclid 1 as

If $A \neq B$ then there exists $C$ with $\mathbf{B}(A, B, C)$.

Tarski postulated instead that every line $A B$ can be extended by the amount $C D$; that is, there exists a point $E$ such that $\mathbf{B}(A, B, E) \wedge B E=C D$. Since Euclid's lines have distinct endpoints, it should be required that both $A \neq B$ and $C \neq D$.

There is an intermediate form we call localextension, in which you are allowed to extend $A B$ by the amount $B C$; that is, the segment used to measure the extension and the segment to be extended have a common endpoint.

Euclid's Prop. I. 2 asserts that given any point $A$ and line $C D$ there is a point $E$ with $A E=C D$. The Tarski extension postulate renders Prop. I.2 superfluous. That is a matter of some regret, since I.2 has a beautiful proof. Clearly the Tarski extension postulate goes beyond what Euclid had in mind; therefore we assume only Euclid 1 as stated above.

The line-circle axiom says that if $P$ is inside circle $C$ then any line through $P$ meets $C$. This axiom is discussed fully in Section 6.10 below, but it is relevant to line extensions,

\footnotetext{
${ }^{14}$ It is discussed by Proclus [38], p. 126, who thinks it superfluous as it is included in the meaning of Postulate 1. Apparently Simson was not convinced, as his translation [17] lists it as an axiom. Heath rejects it as an axiom (p. 232 of [18]), not on mathematical grounds, but because he came to the conclusion that it is an "interpolation", i.e., is not in the original Euclid, in spite of being included in three of the "best manuscripts." ${ }^{15}$ Outer connectivity was discussed already by Proclus, who stated it as "two straight lines cannot have a common segment" [38], p. 168-9, Section 216-17. Proclus says it is implicit in Euclid's line extension axiom. Neverthless, Proclus considers some possible proofs of it-but not the ingenious proof offered by Potts in the commentary to Prop. I.11 in [37], p. 14, which shows that outer connectivity follows from perpendiculars and the fact that an angle cannot be less than itself. The latter, however, is a difficult theorem, if it (or a close equivalent) is not assumed as an axiom.

${ }^{16}$ The Simson translation [17] renders the extension postulate as "That a terminated straight line may be produced to any length in a straight line.” Perhaps Euclid's extension postulate said more than Heath's translation indicates.
} 
because if we have a circle with center at $B$, then line-circle enables us to extend $A B$ by the radius of the circle. In this way one shows that Euclid 2 and line-circle together imply localextension, since the circle needed for a local extension can be drawn with a collapsible compass. With localextension, we can carry out the proof of I.2, and after I. 2 we can prove the Tarski extension principle. From there on the development is unaffected by the choice not to assume the Tarski extension principle as an axiom, but our weaker axiom exactly reflects what Euclid used and permits us to prove I.2 as he did.

The key to this development is the "bootstrapping" aspect of it: one needs I.2 to prove the Tarski extension principle with line-circle, but one needs some extensions by a given amount to prove I.2, so it appears at first that Euclid 1 is not sufficient; so we first prove localextension, then use it to prove I.2, then prove Tarski extension. For this to work it is also necessary that "inside" be correctly defined and that the line-circle axiom be correctly formulated; there are several wrong ways to do these things. Euclid did not give us a complete proof to follow, since he did not state or use any version of line-circle, and did not define "inside" which is the key task in formulating line-circle.

\subsection{Five-line axiom}

Euclid attempted, in Proposition I.4, to prove the side-angle-side criterion for angle congruence (SAS). But his "proof" appeals to the invariance of triangles under rigid motions, about which there is nothing in his axioms, so for centuries it has been recognized that in effect SAS is an axiom, not a theorem.

Instead of SAS itself, we take an axiom known as the "five-line axiom." This axiom is illustrated in Fig. 3. Its conclusion is, in effect, the congruence of triangles $d b c$ and $D B C$ in that figure. Its hypothesis expresses the congruence (equality, in Euclid's phrase) of angles $d b c$ and $D B C$ by means of the congruence of the exterior triangles $a b d$ and $A B D$.

Our version of the five-line axiom was introduced by Tarski, although we have changed non-strict betweenness to strict betweenness. ${ }^{17}$

\subsection{Pasch's axiom}

Pasch [35] introduced the axiom that bears his name, in the form that says that if a line enters a triangle through one side, it must exit through another side (or vertex). That version, of course, is only true in a plane. Seven years later, Peano [36] introduced what are now called "inner Pasch" and "outer Pasch", which work without a dimension axiom. ${ }^{18}$ See Fig. 4. In that figure, we use the convention that solid dots indicate points assumed to exist, while an open circle indicates a point that is asserted to exist.

Technically "Pasch's axiom" should be "Pasch's postulate", since it makes an existential assertion, but the terminology is too well-established to change now.

\footnotetext{
${ }^{17}$ The history of this axiom is as follows. The key idea (replacing reasoning about angles by reasoning about congruence of segments) was introduced (in 1904) by J. Mollerup [32]. His system has an axiom closely related to the 5-line axiom, and easily proved equivalent. Tarski's version [43], however, is slightly simpler in formulation. Mollerup (without comment) gives a reference to Veronese [44]. Veronese does have a theorem (on page 241) with the same diagram as the 5-line axiom, and closely related, but he does not suggest an axiom related to this diagram.

${ }^{18}$ Axiom XIII in [36] is outer Pasch, with $\mathbf{B}(a, b, c)$ written as $b \in a c$. Axiom XIV is inner Pasch. Peano wrote everything in formal symbols only, and eventually bought his own printing press to print his books himself.
} 

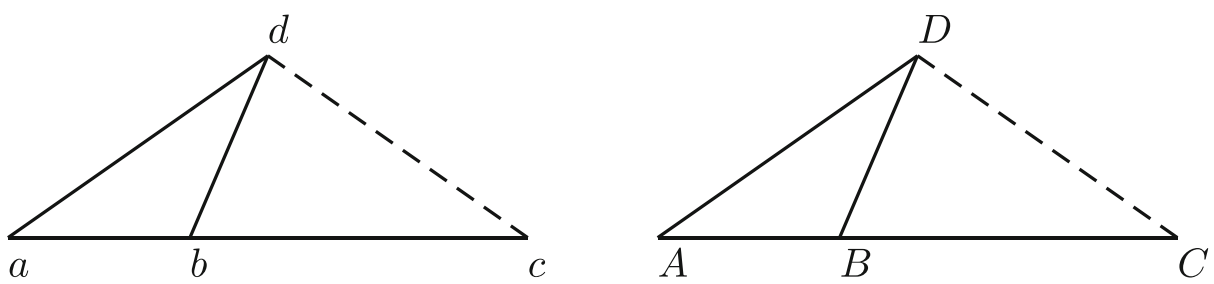

Fig. 3 If the four solid lines on the left are equal to the corresponding solid lines on the right, then the dashed lines are also equal

\subsection{Degenerate cases}

Tarski was always interested in minimizing the number of axioms, and was happy if allowing "degenerate" cases in axioms allowed one to combine what would otherwise be several separate axioms. Tarski's versions of Pasch's axiom allow all the points to lie on one line. From those degenerate cases one can derive basic principles about the order of points on a line that we take as separate axioms. Our reason for requiring a non-collinearity hypothesis in inner and out Pasch is the principle that axioms should correspond to intuition. If you have to draw a different picture to convince yourself that the degenerate case is valid, then that is a different intuition; so generally it should be a different axiom.

Another kind of "degenerate case" is the so-called "null segment" $A A$. In Euclid, lines are given by two distinct points, so there is no such thing as the "line" (or segment) $A A$. Formally we have the predicate $E(A, B, C, D)$. What happens then when $A=B$ ? The idea that $E(A, B, C, D)$ means that line $A B$ is equal (congruent) to line $C D$ suggests that it should be false when $A=B$, since line $A A$ does not exist. But the idea that $E(A, B, C, D)$ means "equidistance" suggests that $E(A, A, C, D)$ should be equivalent to $C=D$. Are null segments allowed, or not? If they are, we need the axiom $E(A, A, C, C)$ that says "all null segments are equal". If they are not, we need $\neg E(A, B, C, C)$. Euclid gives us no guidance: he only works with lines that have distinct endpoints (no null segments), but he never says a single word about null segments. We were (eventually) able to follow Euclid in that respect: we have no axioms either way about null segments, and our axiom system has models with null segments and models without null segments. We are "agnostic" about null segments.

It is not a fundamental philosophical issue, as the talk of "null segments" is just shorthand; nobody suggests that $A A$ is really a line. The argument against null segments is
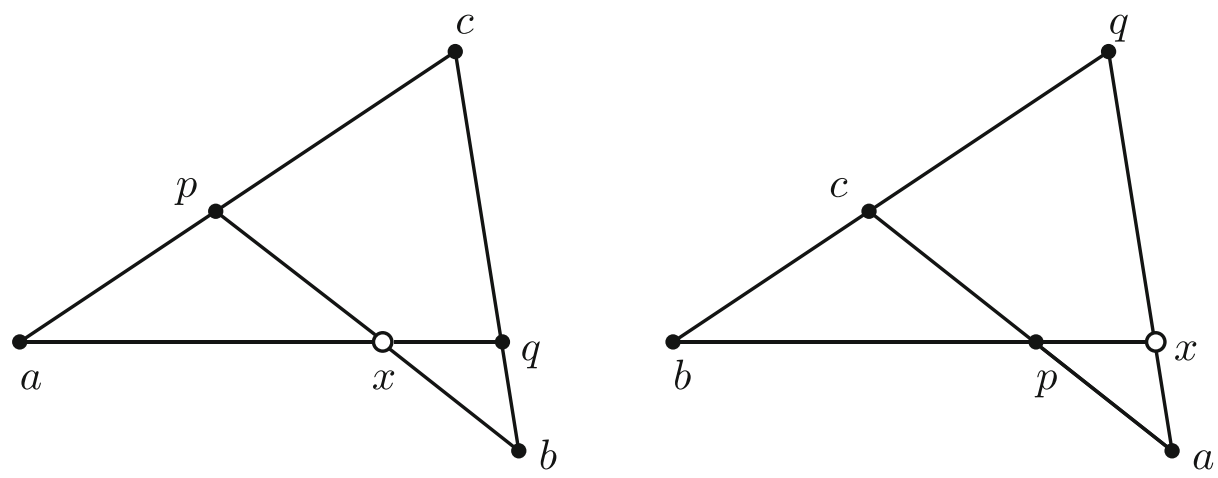

Fig. 4 Inner Pasch (left) and outer Pasch (right). Line $p b$ meets triangle $a c q$ in one side $a c$, and meets an extension of side $c q$. Then it also meets the third side $a q$. The open circles show the points asserted to exist 
that we want to follow Euclid closely. The argument for null segments is that allowing $E(A, A, C, C)$ is occasionally convenient in allowing the succinct statement of theorems. For example, congruence is preserved under reflection in a point, which is called pointreflectionisometry. That is stated by saying that if $B$ is the midpoint of both $A C$ and $P Q$, then $A P=C Q$. That statement includes the case when $A=P$ : then the conclusion is $Q=C$. To keep our theory agnostic, we had to assume $A \neq B$ in pointreflectionisometry, which complicated the proof of linereflectionisometry. (These theorems do not occur in Euclid anyway, but we need them to prove Euclid's Postulate 4, all right angles are equal.)

The picture of the 5-line axiom illustrates that it is meant to capture the intuition leading to the SAS congruence principle. But when the picture collapses onto a line, the 5-line axiom makes "one-dimensional" or "linear" assertions about order and congruence of points on a line. The "degenerate cases" of the 5-line axiom are when point $D$ lies on the line $A B C$. What principles are embodied in those degenerate cases? The cases $D=A$ is sumofparts: if $B(A, B, C)$ and $B(a, b, c)$, and $A B=a b$ and $B C=b c$, then $A C=a c$. The case $D=B$ in the 5-line axiom similarly is differenceofparts: if $B(A, B, C)$ and $B(a, b, c)$, and $A B=a b$ and $A C=a c$, then $B C=b c$. Euclid did not state these principles explicitly, but when he used them, he referred to his common notions about "adding equals to equals" and "subtracting equals from equals".

Following the principle that there should be a one-to-one correspondence between intuitions (or diagrams) and axioms, we should impose the extra hypotheses in the 5-line axiom that $D$ is not collinear with $A C$ and $d$ is not collinear with $a c$. If one does that, one will need to add at least four more "linear" axioms (that are now proved with the help of the 5-line axiom). One needs more than sumofparts and differenceofparts, and we did not discover an ideal set of axioms to add. Moreover, one can argue intuitively for the unmodified axiom as follows: when the fourth point is not on the line, the intuition for the axiom is SAS. But now think of the fourth point moving onto the line; if it approaches a limit on the line, all the quantities mentioned vary continously, so the congruences in the 5-line axiom remain true in the limit. The unmodified axiom makes proofs using it insensitive to the distinction whether point $D$ is or is not on line $A C$, and hence it supports intuitionistic proofs, which a restricted version would not. For these reasons, we retained the unrestricted 5-line axiom.

\subsection{Euclid's postulate 5}

Euclid's "parallel postulate", or "Euclid 5", is a postulate rather than an axiom, because it asserts that two lines meet, i.e., there exists a point on both lines. The hypothesis as Euclid stated the postulate involves angles. We use instead a "points-only" version, illustrated in Fig. 5. Then Euclid's version becomes a theorem.

Most modern geometry textbooks replace Euclid 5 by "Playfair's axiom" (introduced by Playfair in 1729), which asserts the uniqueness of a line parallel to a given line $A B$, through a point $P$ not collinear with $A B$. This also becomes a theorem in our development. Although it does not occur as a proposition in Euclid, it is several times used implicitly in Euclid's proofs. ${ }^{19}$

\footnotetext{
${ }^{19}$ Explicitly: Playfair is used directly in propositions 44, 45, 47, and indirectly in $37,38,42$, 46; and more indirectly in 39, 40, 41, 42, 48; so overall it is used in 39-48 except 43. Euclid 5 is used directly in 29, 39, 42, 44 and indirectly in 29-48 except 31 , which is the existence of the parallel line. Euclid should have proved 31 before 29, to emphasize that Euclid 5 is not needed for it.
} 


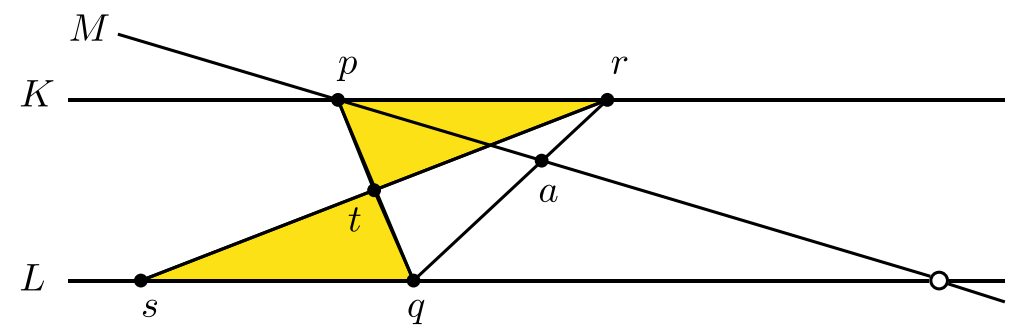

Fig. 5 Euclid 5. Transversal $p q$ of lines $M$ and $L$ makes corresponding interior angles less than two right angles, as witnessed by $a$. The shaded triangles are assumed congruent. Then $M$ meets $L$ as indicated by the open circle

\subsection{Euclid's postulate 4}

Euclid 4 says "all right angles are equal." The definition of a right angle is this: $A B C$ is a right angle if there is a point $D$ such that $\mathbf{B}(A, B, D)$ and $A B=D B$ and $A C=D C$. It has been claimed since the time of Proclus that Euclid 4 is provable, but since the axioms and definitions were not so precise, we are not certain that any of the alleged proofs could be counted as correct until the proof in Tarski's system presented in [40]. In our system this is a difficult proof, depending on the fact that both reflection in a point and reflection in a line are isometries (preserve congruence and betweenness). The proof has to work without a dimension axiom. It is a very beautiful proof and obviously much deeper than the "proofs" given by Proclus and Hilbert. The beautiful part of the proof (after the observation that reflections are isometries) is contained in Satz 10.15 of [40].

Even though this proof is difficult, it would clearly be a flaw to assume Euclid 4 as an axiom, when it can in fact be proved. Therefore we prove it, rather than assume it. ${ }^{20}$

\subsection{Circle construction axioms}

To express our theory in first-order predicate calculus, we use a two-sorted predicate calculus, one sort for points and one for circles. $C I(J, A, B, C)$ means that circle $J$ is a circle with center $A$ and radius $B C$.

Euclid's Postulate 3 is "To describe a circle with any centre and distance." By this, he meant that you can draw a circle with a given center and passing through a given point. This is often called the "collapsible compass" construction, as opposed to the "movable compass" or "rigid compass", that permits drawing a circle with given center and radius specified by a given line (which need not have the center as an endpoint). Euclid's Prop. I.2 shows that the collapsible compass can imitate the movable compass. Past formal systems could not capture the difference. But in our system, Euclid 3 is directly rendered in our language as

$$
A \neq B \rightarrow \exists J C I(J, A, A, B)
$$

\footnotetext{
${ }^{20}$ One may well ask, if we find it necessary to prove Euclid 4 "just because we can", why do we not find it necessary to prove one of the two Pasch axioms, inner and outer Pasch, from the other "just because we can"? The answer is that we still need one of them as an axiom, and the same intuition that justifies one of them also justifies the other. Therefore there is no conceptual economy in reducing the number of axioms by one. But proving Euclid 4 does offer a conceptual simplification.
} 
while the movable compass is

$$
B \neq C \rightarrow \exists J C I(J, A, B, C) .
$$

But a priori, $J$ might also have center $P$ and radius $Q R$. That this is not the case is the content of Euclid's Prop. III.1. The statement of III.1 is "To find the centre of a given circle", but the proof proceeds by showing that two supposedly different centers must in fact coincide. In our formalism that is stated: if $C I(J, A, B, C)$ and $C I(J, a, b, c)$ then $A=a$. (It can be proved that the radii are equal too: $B C=b c$.)

We define $o n(P, J)$ to mean that for some $A, B$, and $C, J$ is a circle with center $A$ and radius $B C$, and $A P=B C$. We do not want a circle to be just a triple of points. That a circle is determined by its center and radius is expressed by saying that if $J$ is a circle with center $A$ and radius $B C$, and $P$ is on $J$, then $A P=B C$. This axiom is called circle-center-radius; see the listing of axioms in an Appendix for a formal statement.

The predicates "inside" and "outside" can be defined using inequality of finite lines, and the circle-circle continuity axiom can be translated straightforwardly from the informal English version given above. We then define relations "on" and "inside" and "outside" that take both a point argument and a circle argument.

\subsection{Circle continuity axioms}

Euclid had no postulates or axioms about circles other than Euclid 3. There are three continuity axioms in the literature:

Circle-circle: if circle $C$ has one point inside circle $K$ and one point outside, then there is a point on both circles.

Line-circle: if line $L$ has a point $P$ inside circle $K$, then there are two points $A$ and $B$ on both $L$ and $K$, such that $P$ is between $A$ and $B$.

Segment-circle: if line $L$ has a point $A$ inside circle $K$ and a point $B$ outside, then there is a point on $K$ between $A$ and $B$.

The reader should bear in mind that in the absence of any dimension axioms, a "circle" is "really a sphere", or even some kind of "hyper-sphere".

Circle-circle is used twice in Book I, once in Prop. I.1 and once again in Prop. I.22, which shows how to construct a triangle with sides congruent to given lines. (The third vertex is the intersection point of two circles with the specified radii.) Although Euclid does not explicitly mention the axiom, both its applicability and necessity are clear, so we take circle-circle as an axiom.

Line-circle is used only twice in Book I, in Prop. I.2 and Prop. I.12, the construction of a "dropped perpendicular." We might also consider "one-point line-circle", in which the conclusion is weakened to assert only the existence of a single point common to $L$ and $K$. Since this axiom is inadequate for the application to I.12, we do not consider further the idea of using it instead of line-circle.

Segment-circle has been suggested as an axiom by many authors, including Tarski (see [43]). But a detailed study shows that it is inadequate; an irremovable circularity arises in formalizing Euclid without a dimension axiom. If we try to construct dropped perpendiculars (Euclid I.12) using segment-circle continuity, to check the hypotheses we need the triangle inequality (I.20). But I.19 is needed for I.20, and I.7 for I.19. In Prop. I.7, the two triangles that are supposed to coincide might lie in different planes, but for the hypothesis that they lie on the same side of a line, a hypothesis that Euclid stated but never used. 
(He could not have used it, since he never defined "same side.") Even so, I.7 is more difficult to prove than Euclid thought, since he took for granted the fact that an angle cannot be less than itself, but that principle is actually the essential content of I.7. Ever since Hilbert [25], angle inequality has been regarded as a defined concept, and proving I.7 then requires dropped perpendiculars (I.12) (or at least, we could not do without I.12). But this is circular. The conclusion is that segment-circle continuity is not a suitable axiom to use in formalizing Euclid's proofs. ${ }^{21}$

The fact is that each of line-circle and circle-circle implies the other, in the presence of the other axioms of Euclid. In the interest of following Euclid fairly closely, we simply take both as axioms: circle-circle is used in I.1 and I.22 (triangle construction), while linecircle is used in I.12, and both those proofs are far simpler than the proofs of line-circle and circle-circle from each other.

Even though we take both as axioms, we remark on the equivalence proofs. The proofs can be found in [24]; see also the last section of [2]. The proof of line-circle from circlecircle relies on dropped perpendiculars, which in Euclid is I.12, proved from line-circle. Therefore, a proof of line-circle from circle-circle must rely instead on Gupta's circlefree perpendicular construction [23, 40], carrying us far beyond Euclid. The only known synthetic proof of circle-circle from line-circle uses the "radical axis" [42].

\subsection{What was Euclid thinking?}

It seems strange that Euclid, who was generally careful, glaringly omits both line-circle and circle-circle. When he needs to use line-circle in the proof of I.2, he instead says "Let the straight line $A E$ be produced in a straight line with $D A$ ". In other words, "let $D A$ be extended until it meets the circle at $E$." Remember that lines are always finite, so line-circle intuitively says that a line can be extended until it meets the circle, as well as saying that (when it is long enough to reach the circle) it cannot pass through the circle at some "missing point" without touching. Probably Euclid thought the difficulty was getting the lines long enough, not getting the circle impenetrable. Then he probably had line-circle in mind when stating Euclid 2, "To produce a finite straight line continuously in a straight line", not just "by some amount", and not "by an amount equal to a given segment", but "enough to meet a given circle", if the starting point is inside that circle.

\section{Equal figures in Euclid}

Euclid defined the word "figure" to mean "that which is contained by a boundary or boundaries", and explicitly mentioned that a circle counts as a figure (so boundaries can be curved). But in Book I, figures are triangles and quadrilaterals, so we do not need to introduce a new primitive sort of variables for "figure." Euclid used the word "equal" to denote a relation between figures that he does not define. One possible interpretation is that equal figures are figures with the same area. But the word "area" never occurs in Euclid, presumably because Euclid realized that he did not know how to define area. Thus, instead of

\footnotetext{
${ }^{21}$ Line-circle continuity does not suffer from this problem, as the triangle inequality is not required to drop perpendiculars. Of course, as Gupta showed [23], one can construct dropped perpendiculars without mentioning circles at all, so there is no formal result that one circle axiom is better for I.7 than another, as none at all is actually needed. We merely say that Euclid's proof can be repaired with line-circle, but not with segment-circle.
} 
saying "the area of the whole is the sum of the areas of the parts", Euclid only reasoned about "equal figures", without defining that notion.

Nor did Euclid give any explicit axioms about "equal figures"; he treated these as special cases of the common notions, such as "if equals are added to equals, the results are equal", where the "addition" of figures refers to what we would call the union of disjoint sets. Occasionally he uses without explicit mention a few further axioms, such as "halves of equals are equal."

Book I culminates in the Pythagorean theorem, which Euclid states using the notion of equal figures. ${ }^{22}$ Although we formalized only Book I in the work reported here, all the propositions in Book II are about "equal figures", so a correct formulation of the notion is critical.

Three ways to make Euclid's notion of "equal figures" precise have been proposed in the past. First, one can define two figures to be equal if they have equal areas. That is not a first-order notion, no matter how area is defined, because it involves measuring areas by numbers. Second, after introducing "segment arithmetic" geometrically, one can define area geometrically, but that is a very great deviation from the path taken by Euclid.

A third interpretation of "equal figures" is the notion of "equal content", explained on p. 197 of [24], which involves cutting figures into a finite number of pieces and reassembling them. That is also not a first-order notion, because of the "finite number of pieces" part. Hence it is irrelevant for our purposes, and we need not go into the details of the definition.

Conclusion: the definitions of "equal figure" that we find in the literature all suffer from one of the following defects:

(i) Not being first order, because of requiring the concept of real number.

(ii) Not being first order, because of requiring the concept of natural number (even just for equality of triangle and quadrilaterals).

(iii) First order, but requiring the geometrical definition of coordinates and arithmetic (addition and multiplication of finite lines), which goes well beyond Euclid.

The introduction of geometrical arithmetic has already been proof checked in [11], from Tarski's axioms; and we checked that Tarski's axioms are equivalent to those used in this paper, so approach (iii) has already been proof checked.

We are, of course, not the first ones to face these difficulties. Hartshorne lists (p. 196 of [24]) the properties of "equal figures" that Euclid's proofs use. Not all the properties in that list are first order. Our approach to the treatment of "equal figures" is to treat "equal triangles" and "equal figures" (that is, equal quadrilaterals) as primitive relations, and give first-order axioms for them, sufficient to account for Euclid's proofs. These axioms are first order versions of Hartshorne's.

Since we use variables only for points, not for figures, we must use two relations: ET for "equal triangles" and EF for "equal quadrilaterals" or "equal figures"; it is only for quadrilaterals, but $\mathrm{EQ}$ is already taken. The axioms for these two relations say that ET and EF are equivalence relations; that the order of vertices can be cyclically permuted or reversed, preserving equality; that congruent triangles are equal; that if we cut equal triangles off of equal quadrilaterals, producing triangles, the results are equal; or if the cuts produce quadrilaterals, the results are also equal. Then we have "paste" axioms that allow for pasting

\footnotetext{
${ }^{22}$ In fact, he says that the squares on the sides together equal the square on the hypotenuse. But what he proves is that the square on the hypotenuse can be divided into two rectangles, each of which is equal the square on one of the sides; so the further notion of two figures together being equal to a third is not really needed.
} 
equal triangles onto equal triangles; if the results are quadrilaterals they are equal, provided also that the triangles do not overlap, which can be ensured by a hypothesis about vertices lying on opposite sides of the paste-line. Figure 6 illustrates one of these axioms. Similarly, if pasting equal triangles onto equal quadrilaterals produces quadrilaterals, they are equal.

Finally we need an axiom that enables us to prove that certain figures are not equal; all the axioms mentioned so far hold if all figures are equal. Such an axiom was introduced by de Zolt (see [24], p. 201). But de Zolt's formulation is not first order. Instead we take a special case: if $A B C$ is a triangle, and $D E$ is a line that cuts the triangle (in the sense that $\mathbf{B}(A, D, B)$ and $\mathbf{B}(B, E, C))$, then neither of the two resulting pieces $A D E$ or $C D E$ is equal to $A B C$ ). This turns out to be sufficient. A complete list of our axioms, including all the equal-figures axioms, is in the Appendix.

\section{Book Zero and filling in book I}

We proved more than 230 theorems, including the 48 propositions of Book I. To list these theorems in the format used in the Appendix requires 14 pages, and since the files containing these theorems are accessible (as well as the proofs), we elected not to list them all. Still we wish to give the reader some idea of the additional theorems that we had to supply. We use the phrase "Book Zero" informally to encompass those theorems that seem to come before Book I, in the sense of being used in Book I and not depending on Book I themselves. Book Zero begins with properties of congruence and betweenness; several important and often-used lemmas are about the order of four points on a line, when two betweenness relations are known between them. (There is one axiom about that, and the rest of the relevant propositions can be proved.) There are variations on the 5-line axiom; there are theorems about collinearity and non-collinearity; there is the definition of "less than" for finite lines, and the ordering properties of that relation and how it respects congruence (or equality) of finite lines. Lying on ray $A B$ (which emanates from $A$ and passes through $B$ ) is a defined relation; there are lemmas about how it relates to betweenness and to collinearity. We can "lay off" a finite line along a ray, and the result is unique. Euclid says we can "add equals to equals" as a common notion; the formal version of this is sumofparts, which as we discussed above can either be an axiom or lemma, depending which version of the 5-line axiom we take. There is also differenceofparts and subtractequals. Equality and order of angles are defined concepts, and we have to prove their fundamental properties in
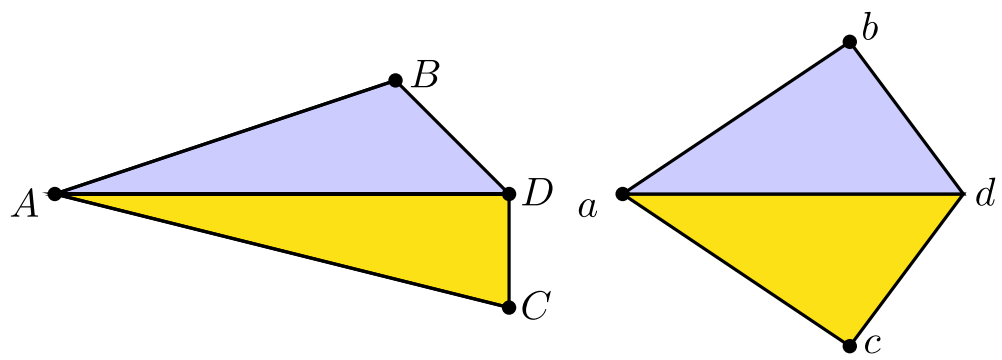

Fig. 6 The axiom paste3. If the lower triangles are equal and the upper triangles are equal, then the quadrilaterals are equal 
lemmas such as ABCequalsCBA, equalanglestransitive, and so on. The "crossbar theorem" is also part of Book Zero. The notions of "same side" and "opposite side" are defined, and their fundamental properties proved, including the plane separation theorem, according to which if $C$ and $D$ are on the same side of $A B$, and $D$ and $E$ are on opposite sides of $A B$, then $C$ and $E$ are on opposite sides of $A B$; that is, there is a point collinear with $A B$ that is between $C$ and $E$. This is where we pass out of Book Zero, however, since the proof of that theorem requires constructing a midpoint, which is Proposition 10.

Book Zero comprises about seventy theorems; Euclid's Book I has 48; we proved an additional hundred of so theorems that are needed to prove Euclid's 48, or are variants of those propositions, but whose proofs use some of Euclid's propositions as well. Let us give a typical example: the lemma we call collinearbetween is used 19 times in our development, including in Propositions 27, 30, 32, 35, 44, and 47. That lemma says that if two lines $A B$ and $C D$ are parallel, and there is a point $E$ between $A$ and $D$ that is also collinear with $B C$, then that point $E$ is actually between $B$ and $C$. That is not trivial to prove, and Euclid simply assumes that it is so, because it appears so in the diagram. The names of some other lemmas will be illustrative:

droppedperpendicularunique, angleordertransitive,

angleorderrespectscongruence, angletrichotomy.

Euclid's proof of angle bisection in I.9, via I.1, cannot be corrected, and instead we prove I.10 (line bisection) using Gupta's 1965 proof, and then prove I.9. Prop. I.12 (dropped perpendicular) has to precede Prop. I.7 (angle bisection), because Euclid's proof of I.7 is hopelessly inadequate, and we give a much more complicated proof that requires perpendiculars. Once perpendiculars are available, we prove pointreflectionisometry and linereflectionisometry, and use them to carry out Szmielew's proof of Euclid's Postulate 4 (all right angles are equal). Euclid fails to state legsmallerhypotenuse, which is needed to prove another fact about right triangles that Euclid uses without proof: the foot of the perpendicular from the right angle to the hypotenuse actually lies between the two endpoints of the hypotenuse. Towards the end of Book I, the steps of the proofs are more cavalier, and the omitted lemmas are more difficult; for example Euclid omitted to state and prove that a square is a parallelogram. ${ }^{23}$

\section{Formal representation of Euclid}

We wanted to write down our axioms, definitions, postulates, lemmas, and propositions in a form that would be easy to manipulate by computer, and independent of any particular computer language, so as to still be readable decades or centuries hence. We chose to use strings to represent all these things. Euclid used only one-character variable names, and we did the same. In that case there seemed no need for commas and parentheses; in other words we used Polish notation. There were, however, more than 26 relations to consider, so we used in all cases two-character names for the relations. For example, we write $\mathbf{B}(a, b, c)$ in the form BEabc. $A B=C D$ is written EEABCD. We used EQAB to represent $A=B$, so we could not use EQ a second time, and chose EE instead. "Parallel" becomes PR, as in PRABCD. There are quite a few of these abbreviated two-character names, but that is enough

\footnotetext{
${ }^{23} \mathrm{~A}$ square has four right angles and equal sides. A parallelogram has both pairs of opposite sides parallel. But there is also this difficulty: Nothing in the definition of a square requires the four sides to lie in the same plane. That can be (and hence should be) proved. We discussed the definition of "parallel" in Section 4.
} 
to convey the idea. The point is that every formula is a string. Conjunctions and disjunctions begin with AN or OR and the subformulas are separated by + or $\mid$, respectively. Negations are formed with NO.

Then we define classes Axiom, Definition, and Theorem, each of which has fields called label, hypothesis, conclusion, and existential. The label field is used for the name. The hypothesis and conclusion fields each contain an array of formulas, or a single formula. The existential field contains an empty string by default, and if it isn't empty, that means that it contains a list of variables that are supposed to be existentially quantified in the conclusion.

We have thus defined a subset of first-order predicate logic. Specifically, our formulas have no function symbols, and only existential quantifiers; universal quantification over the free variables is left implicit. Nested quantifiers do not occur. Polish notation, one-character variables, and two-character predicate names make it easy to manipulate these formulas as strings and arrays of strings, and substitutions can be coded as arrays, making unification possible by regular-expression matching. Every modern programming language has useful libraries for this sort of thing.

\section{Formal proofs}

Each proof is a list of lines. Each line contains a formula and optionally a justification. The proof is kept in a .prf file whose name gives the label of the theorem it is intended to prove. The proof begins with a sequence of unjustified lines that must repeat the hypotheses of the theorem. It ends with a line that is the conjunction of the conclusions of the theorem, or the sole conclusion if there is only one. The first line after the hypotheses must have a justification. Any unjustified lines in the rest of the proof must either be repetitions of earlier lines or must follow by logic alone from some earlier lines. Justifications follow the pattern kind:label, where kind is either defn, axiom, postulate, proposition, or lemma, and label is the label of an item of the specified kind. An axiom, postulate, or definition can be used anywhere, but a lemma or proposition cannot be used anywhere, because circular arguments must be prevented.

We avoid circularity by having a "master list" of lemmas and propositions, which are to be proved in the specified order. A valid proof (of a certain item in the master list) is only allowed to reference previous items in its justified steps.

Euclid's proofs, and ours, make use of arguments by contradiction and cases. We will now explain the syntax we used. An argument by contradiction is introduced by a line with the justification assumption. After some steps of proof, this line must be matched by a line with the justification reductio. This line must contradict the assumption line. By saying that $A$ and $B$ contradict each other, we mean that one of them is the negation of the other. We found it helpful to indent the lines between the assumption and the reductio labels, especially when nested arguments by cases or reductio occur.

The syntax for cases and proof by contradiction is as illustrated in Fig. 7. (The example chosen is the proof of Playfair's form of the parallel postulate, discussed in Section 6.7.) The first two lines state the hypothesis, that we have two lines $C D$ and $C E$ through $C$, both parallel to $A B$. The last line states the desired conclusion, that $C, D$, and $E$ are collinear, i.e., the two parallel lines coincide. In the proof, BE means "between" and CR means "crosses", in the sense given in the line labeled defn: cross.

We wrote more than two hundred thirty formal proofs in this syntax. For convenience, we introduce a name for this subset of first-order logic: Euc, the first three letters of Euclid. 
Fig. 7 An illustrative proof, showing the syntax of formulas and proofs

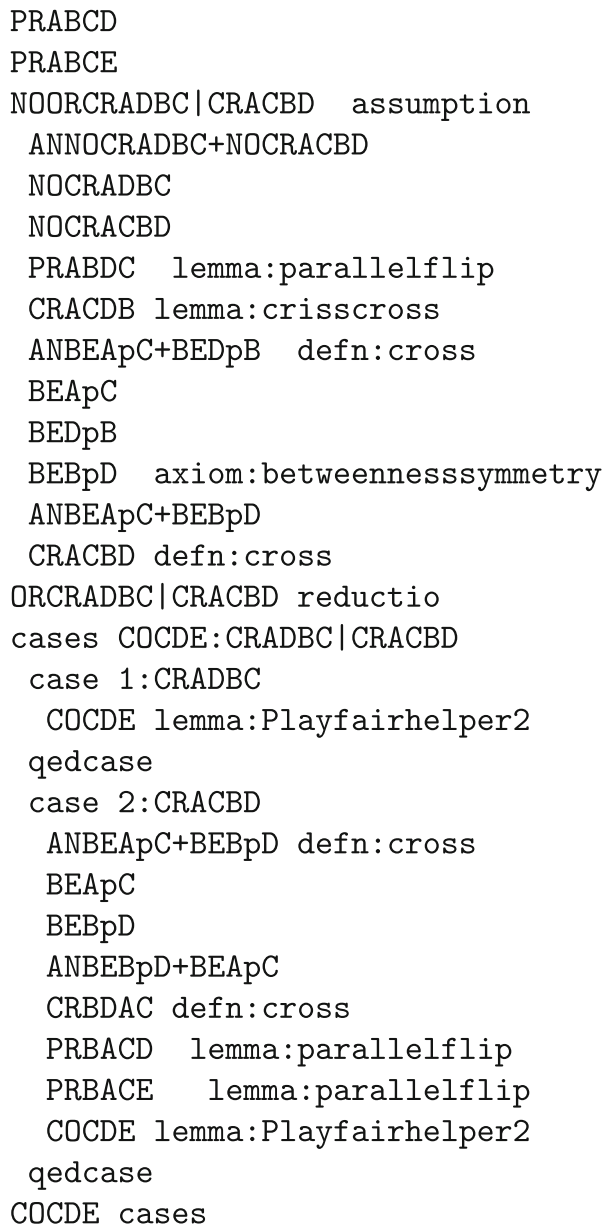

We believe that these proofs will be readable, and computer-checkable, into the indefinite future; in particular, past the lifetime of the currently popular proof assistants that we used to check the correctness of these proofs.

Polish notation is not the favorite of some people, including our referee. But its durability over a long future seems assured-after all it is already more than a century old. We are sure that the scientists of the future will easily be able to add parentheses and commas if they prefer to look at the formulas with those additions; of course they could equally easily strip out the commas and parentheses, so we think it is not an important issue. With or without parentheses and commas, we believe a low-level simple formalism is preferable to a highlevel language (for example the Mizar language), because the purpose of these proofs is to permit easy automatic translation into any desired formal language, without the necessity of resurrecting a language used in the distant past. It is not our purpose in this paper to make the proofs easily readable by humans. That subject will be taken up separately; but our intention is again that formal Polish-notation proofs be the starting point of a translation into something that humans like to read. 


\section{Checking the proofs by computer}

The proofs described in this paper only need a rather weak logic. There are no function symbols, and all the statements have a very restricted form. That made it easy to write a custom-built proof checker, or "proof debugger", that we used while developing the formalization. That tool also checks that we stay within the bounds of that logic.

HOL and Coq use an architecture that guarantees a much higher reliability.

This is called the LCF architecture, after the LCF system from the seventies that pioneered the approach. This architecture divides the system in a small kernel (or logical core) and the rest of the code. By the use of abstract datatypes, the correctness of the mathematics is then guaranteed by the correctness of the kernel. Whatever errors the rest of the code of the system may contain, the statements claimed to have been proved will indeed have been proved. In the case of the HOL Light system (one of the incarnations of HOL), the correctness of this kernel has even been formally proved (using the HOL4 system, another incarnation of HOL), which gives an extremely high guarantee that the system will not have any logical errors.

Our procedure for proof-checking Euclid was thus as follows:

- Write formal proofs in the Euc language, simultaneously checking and debugging them with our custom proof debugger.

- Translate these proofs into HOL Light or Coq syntax by means of simple scripts.

- Check the resulting proofs in HOL Light and Coq

Was it necessary to use the Euc language? No, but it is closer to Euclid than either HOL Light or Coq, and allowed us to write the proofs only once, and moreover, has a better chance of being readable a thousand years from now. Was it necessary to write a custom checker or debugger for Euc? Perhaps not, but it facilitated our work flow by separating the writing of the proofs from the use of the two major proof assistants, and by providing very useful error messages in the case of incorrect proofs. Was it necessary to use two proof assistants? No, since each one is perfectly reliable, but we did it anyway.

The devil might ask whether we have lost something by using higher order logic to check first order proofs. While it may appear so at first glance, actually higher order logic itself ensures that we have not. Consider: in higher order logic (both in Coq and HOL Light) we proved that for any type of points, and any predicates satisfying the axioms of Euc, all our theorems are satisfied. In these statements there is a second-order quantification over the predicates used to interpret betweenness, congruence, and the predicates $E T$ and $E F$. In essence we have proved that the axioms hold in any model (of our axioms for geometry). Then by Gödel's completeness theorem, the theorems are actually first order theorems. However, it is a general feature of higher order theorem provers that they do not directly check first-order proofs. Moreover, we used the Leibniz definition of equality, so indeed our translated proofs are not first order. However, we did check the first order proofs directly in our custom proof checker before translating them to higher order logic.

Our debugger also counted the number of inferences. Proofs of more than 200 inferences were not uncommon, but the majority were under 100 inferences.

We also wrote code that analyzed the dependencies between lines of a given proof. This enabled us to identify and eliminate lines that were never subsequently used. We follow Euclid in sometimes repeating previously deduced lines just before applying a proposition, to make it apparent that the required instances of the hypotheses have indeed been derived. These lines, of course, are technically eliminable; but mainly we wanted to eliminate "red herring" lines that were actually irrelevant. The automatic detection of such lines was useful. 


\section{Checking the proofs in HOL light}

To ensure the correctness of the Euclid formalization from the language Euc to HOL Light, we built a very small custom checker on top of HOL Light, and used that to check our work for correctness in HOL as well. The source of this proof checker, proofs.ml, has about a hundred lines, which then is used to check a translation of the formalization into syntax that HOL Light can process of almost twenty thousand lines, michael.ml. This last file is created from the original proof files described above by two small ad hoc scripts, a PHP script called FreekFiles.php and a Perl script called FreekFiles.pl.

In the HOL system, all input (even the proofs) always consists of executable ML source code. The proofs of each lemma in our case is checked by calling a function run_proof (implemented in proofs.ml) on the statement of the lemma and a list of items of a custom datatype called proofstep. The ML definition of this datatype is shown in Fig. 8. The output of this function is a HOL thm, a proved statement. In other words, the ML type of the function that is used here is:

run_proof : term -> proofstep list $->$ thm.

The list of proof steps corresponding to the proof from Fig. 7 is shown in Fig. 9.

In that example some of the choices on how to translate the statements from the proof to the HOL logic can be seen. For instance, we had to decide whether to translate $\mathrm{EQ}$ to the standard HOL equality, or to have it be a custom relation. We chose to make use of the features of standard first order logic with equality, but nothing beyond that. That means that we translated equality to the built-in equality of the logic, and translated $\mathrm{NE}$ identical to $\mathrm{NOEQ}$ and NC identical to NOCO. Therefore the translation does not have predicates $\mathrm{EQ}, \mathrm{NE}$ and NC. As a small optimization, NONC was translated without a double negation.

Within HOL we used an axiomatic approach. That is, we added our axioms to the HOL axioms. That way, we will be verifying that the theorems of Euclid follow from those axioms, rather than (for example) that they are true in $\mathbb{R}^{2}$ or $\mathbb{R}^{n}$. This raised the number of HOL axioms by 36 , from the original 3 to 39 . Before stating these 36 axioms we also added two new primitive types. point and circle, and five new primitive predicates, $\mathrm{BE}, \mathrm{EE}$, CI, ET and EF.

There are some differences between what was taken to be axioms in the original version of the formalization, and what are axioms in the HOL version. The definitions of the predicates were originally axioms, but in the HOL version are actual HOL definitions, with

Fig. 8 The ML datatype used when checking the proofs with HOL

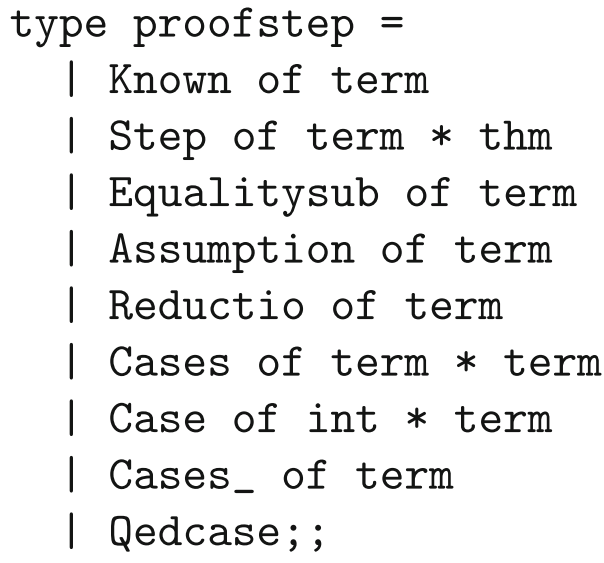




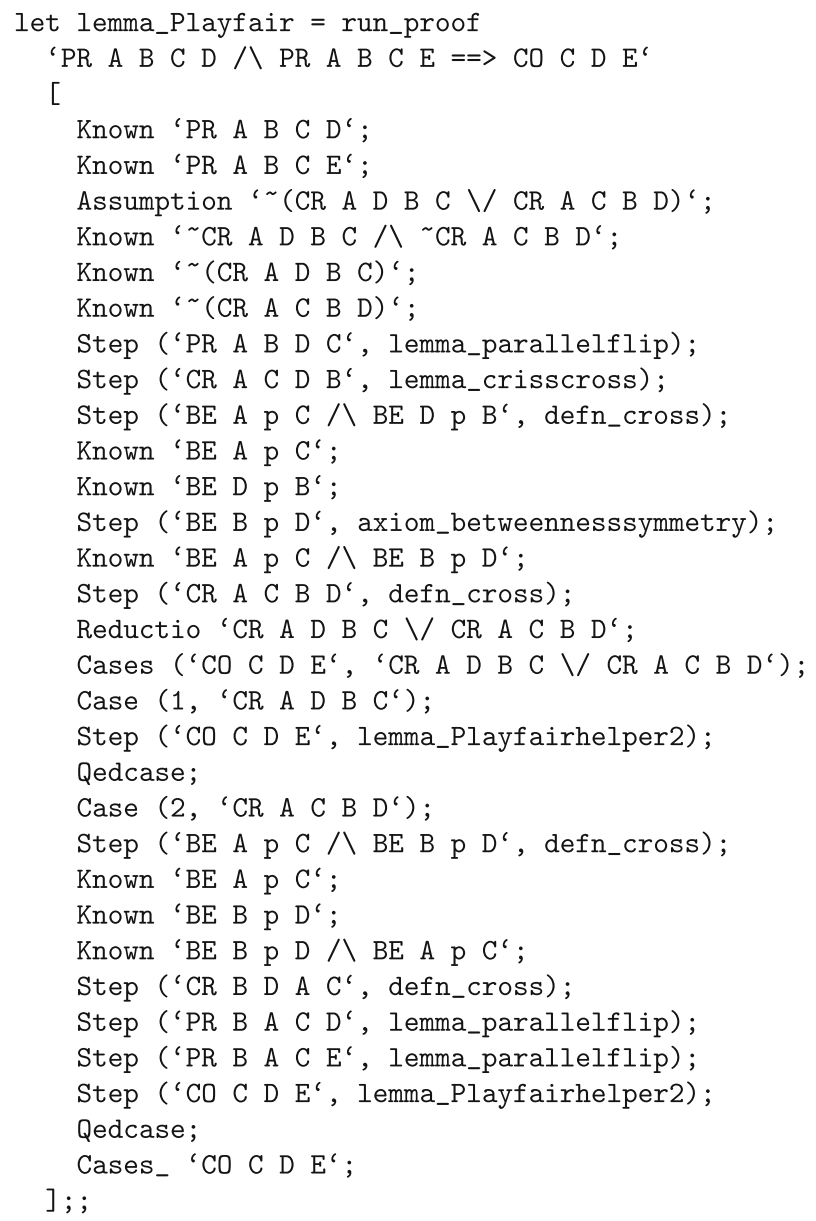

Fig. 9 The HOL counterpart of the proof in Fig. 7

the 'axioms' being the equivalences that these definitions produce. Two exceptions for this approach are defn:unequal and defn:circle of which the first is omitted (it is not used anywhere) and the second is an axiom (as it does not have the shape of a HOL definition). Also, defn:inside, defn:outside and defn: on are still axioms, because these also state that the defining property does not depend on the points that give the circle, which one does not get from just a definition.

Of the common notions, cn: equalitytransitive, $\mathrm{cn}$ : equalityreflexive, cn:stability and cn:equalitysub are not axioms, but proved statements, because they only involve equality and are part of the logic.

The LCF architecture of HOL Light only guarantees that the proofs are valid in the higher order logic of HOL Light. However, the implementation in proof s.ml only uses first order tactics (most notably MESON, which is the main tool used for checking the steps), which shows that the proofs are actually first order. The proofs, written in the Euc language, contain on each line a formula and its justification. HOL Light checks that the formula 
follows from previous steps of the proof, using the axiom, theorem, or definition mentioned in the justification, together with first-order logic. The simple logic associated with Euc allows only instantiation of the axiom, theorem, or definition; or in proofs by cases and contradiction, some simple propositional logic. Is HOL Light then really checking the given proof? Yes, it is, because (a) even if it found a more complicated first-order proof, that would still be a check, and (b) in any case, HOL Light will not use quantificational logic to prove a theorem without quantifiers.

The full check of the twenty thousand lines is uneventful, but not very fast, mostly because of the use of the rather heavy MESON. It takes several minutes. At some points MESON has to work very hard (it loses the fact that the conclusion of the lemma exactly matches the step being proved, and is trying many possible ways to unify the parts). For these cases proofs.ml contains two custom lower level tactics, SUBGOAL_UNFOLD_TAC and SUBGOAL_MATCH_TAC, which do not use MESON.

\section{Checking the proofs in Coq}

\subsection{Formalizing the axioms}

The axioms in our axiomatization of Euclid are of two kinds: axioms related to definitions and others. Axioms that serve as definition as those of the form: $\forall x, P(x) \Longleftrightarrow Q(x)$. We translate them to a proper Coq definition to reduce the number of axioms. Technically, in Coq, we did not use the Axiom keyword. Because axioms are similar to global variables in a programming language, they reduce the re-usability of the code. We sorted the axioms into several groups, and defined them in Coq using type classes. Then, the axioms are given as so-called section variables of Coq, a mechanism which allows to have the axioms as an implicit assumption for each lemma. For propositions I.1 to I.28 and I.30 we do not use the fifth postulate of Euclid. For propositions I.1 to I.34, we do not need the equal-figure axioms. Avoiding the Axiom keyword allows us to reuse the proofs in a different setting by proving the axioms in Coq as a second-order property either from another axiom system or by constructing a model (see Section 14.1).

Equality We model the equality using Coq's built-in equality: Leibniz's equality. We could also have assumed an equivalence relation and substitution properties for each of the predicates of the language.

\subsection{Verifying the proofs}

Proof assistants differ in their mathematical foundations (e.g. type theory, higher order logic (HOL), or set theory) and their proof language. In procedural style proof assistants (e.g. Coq and HOL Light), proofs are described as a sequences of commands that modify the proof state, whereas in proof assistants that use a declarative language (e.g Mizar and Isabelle), the proofs are structured and contain the intermediate assertions that were given by the user and justified by the system.

We wrote a script to translate the proofs to Coq's language. The translation is relatively easy as the proofs steps used by the proof debugger are small.

Our translation generate a proof in the traditional language of Coq, not in the declarative language introduced by Corbineau [16] because this language is not maintained. But, the 
formal proofs we generate are in the declarative style: for case distinctions we give explicitly the statement which is used instead of the name of the hypothesis, the proofs are purely in the forward chaining style, based on sequences of applications of the standard Coq tactic assert. We do not introduce hypothesis numbers. The existential statements and conjunctions are eliminated as soon as they appear by introducing the witness and decomposing the conjunction. The assertions are justified using some ad-hoc tactics which use some automation and congruence closure. We had to circumvent some weakness of Coq's automation. Coq is not able to use efficiently lemmas of the form $\forall x y z, P x y z \rightarrow P y z x \wedge P y x z$, because the apply tactic will always choose to unify the goal with the first term of the conjunction. The standard way to state such a lemma in Coq is to split the lemmas in two parts. But, we did not want to modify the original formalization of the lemmas. Hence, to circumvent this limitation, we verified these proof steps using a tactic based on forward-chaining. The case distinction tactic allows to distinguish cases on previously proved disjunctions or disjunctions which are classical tautologies. The tactic can deal with n-ary disjunctions. The proof script is structured using curly brackets and indentation. Each proof step of the original proof corresponds to one proof step in Coq, except that steps for which there is no justification in the original proofs. Those steps correspond to the natural deduction rule for introduction of implication and are implemented using the standard Coq tactic intro. All tactics are designed such that the names of the geometric objects are preserved from the original proofs but the names of the hypotheses are automatically generated by Coq and not used explicitly in the proofs. Figure 10 displays the proof of Playfair's axiom of uniqueness of parallels in Coq's language enriched by the tactics to ease the verification. Some of the predicates have been renamed into longer names to enhance readability and to match the names used in the GeoCoq library. The proofs are verified using classical logic. The proofs can be checked by Coq in about 90 seconds using an Intel(R) Core(TM) i7-7700 CPU @ 3.60GH with $32 \mathrm{Go}$ RAM.

\subsection{Verifying the statements}

When evaluating a formalization, even if we trust the proof checker, we need to check that statements are formalized faithfully. Usually the only method we can use for this process is to check the statements by human inspection and trust the reviewer to also check the statements. For this formalization, we were lucky, as many statements had been formalized independently by the first author and the GeoCoq team. To improve the confidence in the formalization, we compared the two formalizations of the statements to detect potential defects. We detected only minor differences in some of the statements.

\section{The axioms hold in $\mathbb{R}^{2}$}

The axioms fall into two groups: those that are variants of Tarski's axioms A1-A10, and the equal-figure axioms. In order to make sure that there is no mistake in the axiomatization, we wished to check formally that the axioms hold in the Cartesian plane $\mathbb{R}^{2}$. For reasons of convenience, we checked that for the first group of axioms in Coq, and for the equal-figure axioms in HOL Light. We will discuss these two verifications separately.

It has already been checked [7] that Tarski's axioms hold in $\mathbb{R}^{2}$. In Tarski's A1-A10, without dimension axioms, one can (formally) verify our axioms, which are mainly different from Tarksi's by using strict betweenness and hence avoiding degenerate cases. This has also been verified in Coq. 


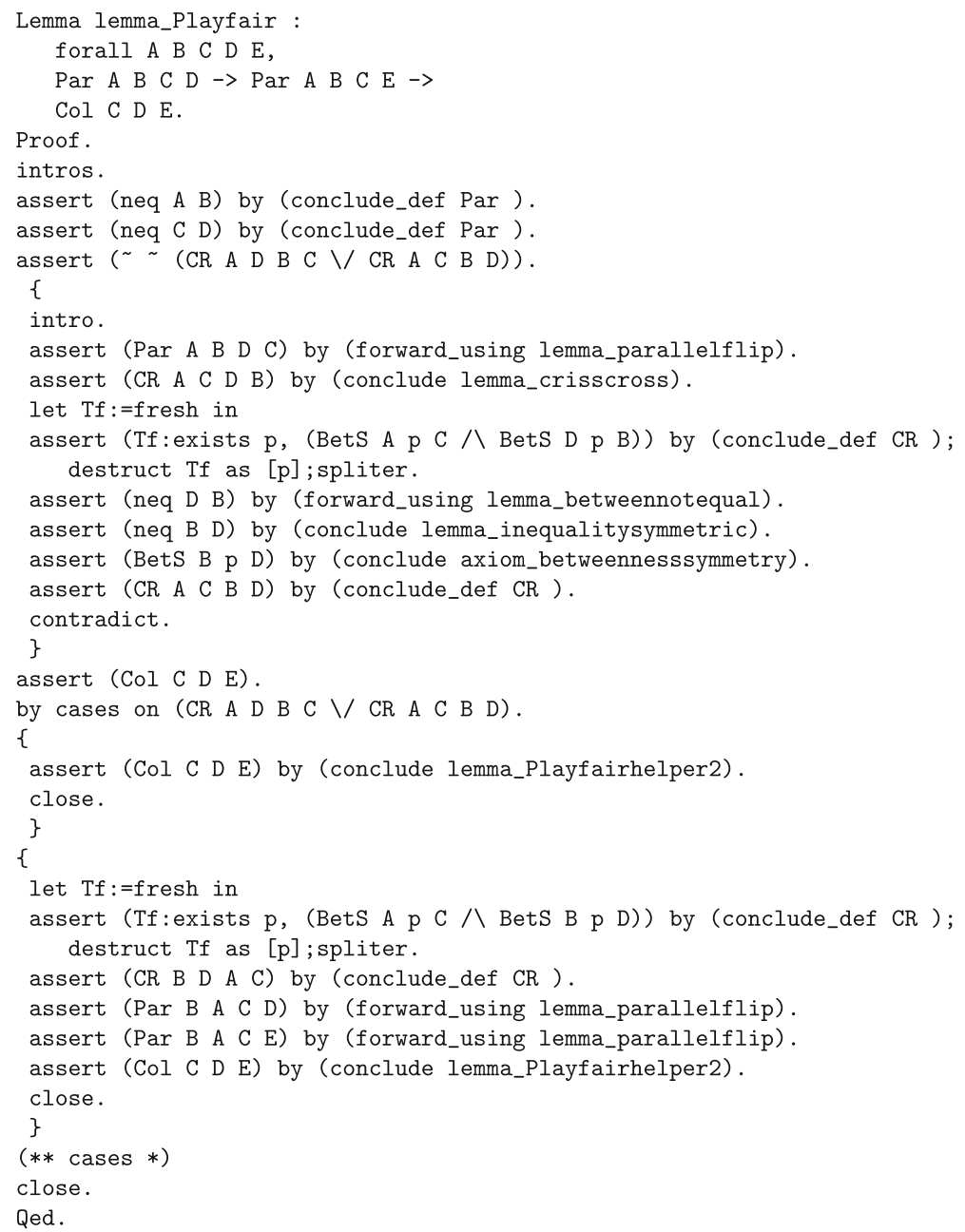

Fig. 10 Example proof in Coq

\subsection{Verifying the Tarski-style axioms in Coq}

Since there is no dimension axiom, the "intended model" is $\mathbb{R}^{n}$, for any integer $n>1$. More generally, we wish to prove that if $\mathbb{F}$ is a Euclidean field, then $\mathbb{F}^{n}$ satisfies our axioms. We break this claim into four parts.

(i) our axioms for neutral geometry can be derived from the corresponding Tarski's axioms.

(ii) circle-circle, circle-line, and Euclid 5 can be derived from the corresponding axioms from Tarski.

(iii) Tarski's axioms hold in $\mathbb{F}^{n}$. By "Tarski's axioms" we mean those of our axioms that expressed using betweenness and equidistance. (These are similar to the axioms and some theorems of Tarski's geometry, except we use strict betweenness.)

(iv) the equal-figure axioms hold in $\mathbb{F}^{n}$. 
The reason for passing through Tarski's axioms is that it has already been shown that Tarski's axioms for neutral geometry hold in $\mathbb{F}^{2}$, when $\mathbb{F}$ is a Pythagorean ordered field. ${ }^{24}$ Specifically, Boutry and Cohen have formalized the proof that the Cartesian plane over a Pythagorean ordered field is a model of our formalization of Tarski's axioms A1-A10 using ssreflect [5].

Ad (ii). We chose to assume the circle-circle intersection property and use the formal proof obtained by Gries and the second author that the circle-line intersection property can be derived from circle-circle intersection, even without assuming a parallel postulate [22]. Gries also formalized the proof that circle-circle intersection can be derived from the continuity axiom of Tarski (Dedekind cuts restricted to first-order definable sets). As Euc axiom system assumes that we have a sort for circles, we need to define the type of circles from Tarski's axioms (and Coq's Calculus of Inductive Constructions CIC). The type of circles is defined as the triple of points ( $*$ is interpreted in this context as the Cartesian product).

Definition Tcircle : Type := Tpoint*Tpoint*Tpoint \%type.

Then the predicate CI can be defined by:

Definition CI (J:TCircle) A C D : = J $=(\mathrm{A}, \mathrm{C}, \mathrm{D}) \quad \bigwedge \mathrm{C}<>\mathrm{D}$.

Then the relation expressing that a point is on a circle can be defined by destructing the triple:

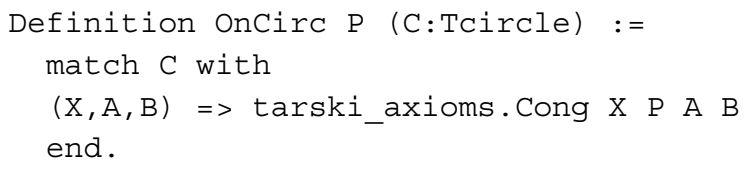

For Euclid 5, we rely on the proofs of equivalence between different versions of the parallel postulates studied previously by the first author [3] and formalized in Coq, by Boutry, Gries, Schreck and the second author [8].

\subsection{Verifying the equal-figure axioms}

We here outline the verification that the equal-figure axioms hold in $\mathbb{R}^{2}$. We did not verify them in $\mathbb{F}^{n}$ or even $\mathbb{R}^{n}$, because we wanted to use the (scalar) cross product in $\mathbb{R}^{2}$ and the existing tools for real and vector algebra in HOL Light.

We interpret points as members of the HOL Light type real^^2. We write that here as $R^{2}$. Then we define the dot product and the two-dimensional cross product as usual:

$$
\begin{aligned}
(a, b) \cdot(c, d) & =a c+b d \\
(a, b) \times(c, d) & =a d-b c
\end{aligned}
$$

Twice the signed area of a triangle $a b c$ is defined by

$$
\operatorname{tarea}(a, b, c)=(c-a) \times(b-a) .
$$

We define ET (equal triangles) by saying that two triangles are equal if the absolute values of their signed areas are equal. That is, $E T(a, b, c, p, q, r)$ means

$$
|\operatorname{tarea}(a, b, c)|=|\operatorname{tarea}(p, q, r)| \text {. }
$$

\footnotetext{
${ }^{24}$ Recall that a Pythagorean field, is a field where sums of squares are squares.
} 
Twice the signed area of a quadrilateral $a b c d$ is given by the cross product of its diagonals:

$$
\operatorname{sarea} 4(a, b, c, d)=(c-a) \times(b-d) .
$$

The area of $a b c d$ is the absolute value of the signed area. We wish to define $E F$ (equal quadrilaterals) by saying two quadrilaterals are equal if the squares of their signed areas are equal. But we do not allow just any four points to be a quadrilateral. Instead we allow two kinds of quadrilateral, convex quadrilaterals and ones which are "really triangles", meaning that one vertex is between two adjacent vertices. We define $a b c d$ to be convex if its diagonals cross, i.e., there is a point $m$ with $\mathbf{B}(a, m, c) \wedge \mathbf{B}(b, m, d)$. In our formal development the condition $0<\operatorname{area}(a, b, c, d)$ is imposed separately. We interpret $E F(a, b, c, d, p, q, r, s)$ to mean that $a b c d$ and pqrs are each quadrilaterals in this sense, and their areas are equal.

Betweenness $B(a, b, c)$ is interpreted (or defined) thus:

$$
B(a, b, c) \leftrightarrow \exists t .(b-a)=(c-a)>0 \wedge 0<t<1
$$

These are all the definitions needed to interpret the equal-figures axioms in $R^{2}$. We executed this translation by hand, producing a list of sixteen goals to prove in HOL Light. These theorems turned out not to be as trivial as we initially thought; the axiom that says congruent triangles are equal required nearly 2000 lines of HOL Light proof. Even more lines were required for the last axiom, paste 4 . Altogether the verification of all 16 equalfigure axioms required about six thousand lines of HOL Light proofs.

Although theoretically these theorems fall within the domain of quantifier elimination for the real field, in practice they have too many variables, and quantifier elimination was not used. Instead, we used rotations and translations to reduce the complexity, and used only the standard vector-algebra theorems that are distributed with HOL Light. Many of the axioms are consequences of the additivity of area, so we proved them by first proving (four or five different forms of) the additivity of area, and then deriving the axioms from the additivity of area. Numerous lemmas seemed to be required. Just to mention two examples:

(i) between_norm, which says that $B(a, b, c)$ is equivalent to the "norm condition",

$$
|b-a|+|c-b|=|c-a| .
$$

(ii) If $a b c d$ is a convex quadrilateral (its diagonals meet), and has positive area, then $a b$ and $c d$ have no point in common.

As mentioned, we used the scalar cross-product to define area. Alternately, we could have used the definition of area by Lebesgue measure, which already exists in the HOL Light library. Since we did not do that, we cannot say for certain that it would not have been easier, but there certainly would have been many details and extra lemmas in that approach as well.

\subsection{An inconsistency and its repair}

Our equal-figure axioms include axioms about cutting and pasting equal figures to get other equal figures. See Fig. 6 for an example, namely paste3, which is about pasting together triangles with a common side $A C$ to get a quadrilateral $A B C D$. This is used in Propositions 35,42 , and 47 . Of course we need something to ensure that the two triangles are in the same plane, and that when pasted together, they do make a quadrilateral. The obvious 
hypothesis is that $B$ and $D$ are on opposite sides of the common side $A C$, and that was our first formulation.

Our equal-figure axioms also include axiom cutof $f 2$, which says that if we cut equal triangles off of equal quadrilaterals, with one end of the cut at a vertex and the other on a non-adjacent side, the results are equal quadrilaterals. This axiom is used only once, in Prop. 43; that step of Euclid's proof is justified by common notion 3, about subtracting equals from equals. We formulated this axiom without any formal statement that the resulting quadrilateral is in fact a quadrilateral; nor does Euclid have any such justification in his proof.

It turned out, as we discovered when attempting to verify that the axioms hold in $\mathbb{R}^{2}$, that these two formulations are inconsistent: This version of paste 3 permits the creation of a non-convex quadrilateral $A B C D$ in which diagonal $B D$ passes outside the quadrilateral. Then when we attempt to cut off a triangle $B A P$ with $P$ on $A D$, the triangle is outside rather than inside, so the area of the result might be larger, rather than smaller, than the area of $A B C D$. This phenomenon leads to an inconsistency.

There are two possible ways to remedy this problem: either strengthen the hypotheses of cutoff 2 (requiring that the resulting quadrilateral have crossing diagonals), or strengthen the hypotheses of paste 3 (requiring that $A B C D$ have diagonals that cross, or meet at $A$ or $C$ ). Either way works. Which is more faithful to Euclid? Since these axioms are used only a few times in Euclid, it is not a matter of much importance. The argument against modifying cutoff 2 is that we would then have to verify the added condition in the proof of Prop. 43, which Euclid evidently felt no need to do, and which would probably double the length of the proof of Prop. 43. The argument against modifying paste 3 is that it might not suffice for a proof that requires constructing a non-convex quadrilateral. But there are no non-convex quadrilaterals in Books I-III, so we chose to modify paste 3 rather than cutoff2. This permits us to apply these axioms in Euclid's proofs without adding more steps.

\section{Previous work on computer checking geometry}

Work on computerizing Euclidean geometry began in 1959, in the first decade of the computer age, with the pioneering work of Gelernter [19, 20]. (The reference has a later date because it is a reprinting in a collection.) The axiom system used by Gelernter was not given explicitly, but from the example proofs given, it can be seen that it was, at least in effect, a points-only system. It was a strong axiom system, including for example all the triangle congruence theorems, Euclid 4, some strong but unspecified betweenness axioms. Tarski is not referenced; that is not surprising as Tarski's first publication of his axiom systems was also in 1959. Gelernter's system was claimed to be as good as "all but the best" high school geometry students. Considering the primitive hardware and software of 1959, it was an amazing program. However, its authors stated that they viewed geometry as just one area in which to study heuristic reasoning, and neither the program nor its underlying formal theory ever raised its head above water again.

As far as we know, nineteen years passed before the next work in computerizing geometry; it was 1978 when Wen-Tsün Wu [45] began a series of papers on the subject, culminating in his 1984 book, published only in Chinese, and not available in English until 1994 [46]. He was soon assisted by S. C. Chou [12, 13]. Using coordinates, one reduces a geometry theorem to an implication between polynomial equations. One can then demonstrate the truth of a geometry theorem by algebraic methods. Such algebraic methods, while 
they may succeed in establishing the validity of a theorem, do not provide a proof from geometric axioms. One might check the correctness of Euclid's results this way, but not his proofs. One problem with this approach is that it works only for problems that involve equality, not for problems that involve betweenness or inequality. A second problem is that Wu's method requires polynomials with hundreds of variables, and hence does not produce human-comprehensible proofs. Nevertheless, Wu and Wang, Gao, Chou, Ko, and Hussain proved many theorems. Within a few years three different groups began to use Gröbner bases to do the algebraic work, instead of the Wu-Ritt algorithm. See [15, 26, 27].

Sometime after 1984, Chou invented the area method, which still uses polynomial computations, but based on certain geometric invariants. The area method, as an algorithm for solving geometry problems, can be used by humans and has even been used to train students for Olympiad-style competitions. [14], p. xi. For the state of the art in area-method implementation as of 1994, see [14], where more than four hundred computer-produced and human-readable proofs are given. However, the steps of these proofs are equations, whose truth is verified by symbolic computation, not by logic. Also, as with Wu's method, inequalities and betweenness cannot be treated.

In the 1980s, Larry Wos experimented with proving geometry theorems in Tarski's theory using the theorem-prover OTTER. Then Art Quaife took up that same project, publishing a paper in 1989 [39], and devoting a chapter of his 1992 book to it. Wos and Quaife left a number of "challenge problems" unsolved by OTTER. In this same decade, the book [40] was published, containing the results from Gupta's thesis [23] and Szmielew's Berkeley course, together constituting a systematic development of "absolute geometry" (no circle or continuity axioms) from Tarski's axioms. This book was quite rigorous, but not (yet) computer-checked. It also did not reach even to the beginning of Euclid. Twenty years later, the first author and Wos returned to this project, and used OTTER to find proofs of all the challenge problems of Quaife, and the first ten chapters of [40] (Part I). But since [40] spends a lot of effort developing "elementary" results from minimal axioms (no circle axioms and postponing the parallel postulate as long as possible), the propositions of Euclid are not reached. In this project, the more difficult proofs were not found automatically, but instead the theorem prover was used almost like a proof checker, by means of supplying "hints." Therefore, when we wanted to proceed to proof check Euclid, it seemed appropriate to switch from a theorem prover to a proof checker, which is what we did for this work.

In 2009, Avigad, Dean, and Mumma [1] reported on a formal system for Euclid's Elements. This system is six-sorted (points, lines, circles, segments, angles, and "areas" (figures), and therefore has also a large number of primitive relations, including "same side" and "opposite side". The axiom system of E differs from ours, because it contains more axioms. They assume 20 constructions axioms, 34 axioms about the two-side, inside and betweenness relations which they call "diagrammatic inferences." Their system is intended for two-dimensional geometry only, a restriction deliberately avoided in our system. They also assume that distances and areas can be measured using a linearly ordered abelian group. We follow Euclid in not assigning measures to distance or area; one may compare distances or areas (figures), but not measure them.

The intention of those authors (see Section 6 of [1]) was to build an interactive proof checker (with application to education). They wanted to separate the "diagrammatic inferences" from the inferences that Euclid wrote out, using an algebraic program for the diagrammatic inferences and a prover for the others. The steps that Euclid omitted would 
be done by algebra instead of by logic. This is a "hybrid" approach, halfway between the completely axiomatic approach and the computer-algebra techniques of verifying geometry theorems by converting them to equations. So far, it has not been made to work, ${ }^{25}$ but even if it did work, we would still prefer the completely axiomatic approach, which is part of the tradition extending all the way from Euclid, through Hilbert and Tarski, to the present.

Starting in 2007, and still continuing as this is written in 2018, the second author of this paper and Gabriel Braun have been busy computer-checking geometrical theorems in the proof assistant Coq. They verified Pappus's theorem [11] (which is important for the geometrical definition of arithmetic). They verified that Hilbert's axioms follow from Tarski's [10]. With Pierre Boutry, they verified that Tarski's axioms follow from Hilbert's [9], and completed [6] the verification of the theorems in Szmielew's part of [40], which the second author began (with other co-authors) in [41]. Work is currently being done toward checking Euclid's propositions from Hilbert/Tarski axioms within Coq. This work differs from the work presented in this paper, because the goal is not to verify Euclid's proofs but Euclid's statements using an axiom system as minimal as possible.

For further information, please see the forthcoming survey article [34].

\section{How wrong was Euclid?}

The point that has given rise to the most discussion when our work has been presented is not whether our proofs are certifiably correct, but whether Euclid's proofs are really wrong, or "how wrong" they are. Therefore we address this issue explicitly. We classify the problems with Euclid's proofs into

- Missing axioms (circle axioms, Pasch, betweenness on a line)

- Gaps (correctable failures to prove collinearity or non-collinearity or betweenness)

- Superfluous axioms: theory of angles (angle equality and order can be defined and its properties proved, rather than assumed).

- Difficult theorems "justified" by common notions. For example, it can be proved that an angle cannot be less than itself. Euclid uses this at the end of I.7 without given any justification at all. Hilbert does no better: he assumes it by including uniqueness in his angle-copying axiom.

- Superfluous axioms: Postulate IV (all right angles are equal) can be proved, though a correct proof is rather difficult.

- $\quad$ Out and out errors. We have mentioned Euclid's incorrect proof of the angle bisection proposition I.9, and his uncorrectable proof of I.7. Euclid's errors, and our correct proofs of his propositions, deserve a full discussion, but that will be lengthy and is postponed to a future publication. Our purpose in this paper is to discuss the proof-checking, not the geometry, i.e., not the proofs themselves.

\footnotetext{
${ }^{25}$ Kenneth Manders has studied the role of diagrams in Euclidean proofs and argue that the use of diagrams in Euclid is limited to some class of properties [28]. Luengo and Mumma have proposed formal systems which intend to capture the obvious spatial properties as built in inference rules [33], but both systems have been shown by Miller to be inconsistent [29, 31]. Miller has proposed another system, but the number of cases which should be considered renders the diagrammatic system difficult to use in practice [30].
} 


\section{Conclusion}

Our aim was to remove every flaw from Euclid's axioms, definitions, postulates, and common notions, and give formal proofs of all the propositions in Book I. Did we achieve that aim?

The statements of the postulates and the definitions needed little if any change; it is the axioms and proofs that needed corrections. We replaced Euclid's axioms and postulates by similar ones in a language similar to Tarski's, but using strict betweenness. We added line-circle and circle-circle axioms, and both inner and outer Pasch; we added the five-line axiom to enable a correct proof of the SAS congruence criterion (Prop. I.2). We dropped Postulate 4 (all right angles are equal) because it can be proved, and formulated Postulate 5 (the parallel postulate) in our points-only language. Inequality of lines and angles, and equality of angles, become defined concepts and the common notions concerning those concepts become theorems. We used Tarski's definition of "same side", an essential concept which Euclid mentioned but neither defined nor considered as a common notion. We used Euclid's rather than Tarski's extension axiom, so that Euclid's I. 2 would not be superfluous. We think that this choice of axioms is very close to Euclid's.

With this choice of axioms, we were able to prove Euclid's propositions I.1 to I.48. These proofs follow Euclid as closely as possible, and have been checked in two well-known and respected proof checkers. We have therefore shown beyond a shadow of a doubt that these proofs are correct. Then we checked, again using those same proof checkers, that the axioms we used hold in $\mathbb{R}^{2}$. In particular all of Euclid's propositions in Book I and corrected proofs of those propositions, close to Euclid's ideas, are valid, without a shadow of a doubt, in Euclidean two-space. ${ }^{26}$ While many paper-and-pencil formalizations of Euclid have been put forward in the past, we are the first to be able to make this claim. That this was not a superfluous exercise is shown by the many difficulties we encountered, and the fact that we had to prove the propositions in quite a different order than Euclid, and in some cases by different proofs. In this paper we have focused on the axioms and the proof checking. A subsequent publication will present the geometrical difficulties and compare our proofs in detail to those of Euclid.

To play the devil's advocate, what argument could be made that we did not achieve the aim stated above? One might complain that we proved the propositions in a different order than Euclid did. We had to do that, because we could not prove them in the original order using our axioms. The devil might argue that we should have strengthened the circle-circle axiom to provide for an intersection point of the two circles on a given side of the line joining the centers. With this stronger axiom we could have fixed Euclid's proof of I.9 (angle bisection) and used it as Euclid did to bisect a line. But this would amount to assuming, rather than proving, the existence of erected perpendiculars, midpoints, and angle bisectors. Besides, it would not have fixed the other problems we had with the ordering of theorems, and the proof we gave, using midpoints to bisect angles rather than bisection of angles to construct midpoints, is beautiful, even if it was discovered by Gupta more than two thousand years after Euclid.

We think that the devil would be wrong to say we should have strengthened circle-circle, and we therefore claim that we did indeed remove every flaw from Euclid's axioms, definitions, postulates, and common notions, and give correct proofs of the propositions in Book I.

Open Access This article is distributed under the terms of the Creative Commons Attribution 4.0 International License (http://creativecommons.org/licenses/by/4.0/), which permits unrestricted use, distribution, and reproduction in any medium, provided you give appropriate credit to the original author(s) and the source, provide a link to the Creative Commons license, and indicate if changes were made.

\footnotetext{
${ }^{26}$ They hold in Euclidean $n$-space too, since there is no dimension axiom, but we did not formally check that.
} 


\section{Appendix A: Formal proof of Prop. I.1}

The reader may compare the following proof to Euclid's. The conclusion ELABC, that $A B C$ is equilateral, is reached about halfway through, and that corresponds to the end of Euclid's proof. The firsts half of our proof corresponds fairly naturally to Euclid's, except for quoting the circle-circle axiom, and verifying that is hypotheses are satisfied.

The last half of our proof is devoted to proving that $A B C$ is a triangle, that is, the three points are not collinear. Note the use of lemma partnotequalwhole. If Euclid had noticed the need to prove that $A B C$ actually is a triangle, he would have justified it using the common notions, applied to equality (congruence) of lines. This version of "the part is not equal to the whole" is not an axiom for us, but a theorem.

At the request of the referee we present this proof in a typeset form rather than in its native Polish form. Obviously further mechanical processing can increase its superficial resemblance to Euclid's style, but the point of our present work is simply its mechanicallychecked correctness.

Proposition A.1 (Prop. I.1) On a given finite straight line to construct an equilateral triangle.

$$
\forall A B \quad A \neq B \rightarrow \exists X \quad A B X \text { is equilateral } \wedge A B X \text { is a triangle }
$$

Proof Let $J$ be such that $J$ is the circle of center $A$ and radius $A B$ by postulate Euclid3.

$B \neq A$ by lemma inequalitysymmetric.

Let $K$ be such that $K$ is the circle of center $B$ and radius $B A$ by postulate Euclid3.

Let $D$ be such that $A$ is strictly between $B$ and $D \wedge A D \cong A B$ by lemma localextension.

$A D \cong B A$ by lemma congruenceflip.

$B A \cong B A$ by common notion congruencereflexive.

$D$ is outside circle $K$ by definition of outside.

$B=B$ by common notion equalityreflexive.

$B$ is inside circle $K$ by definition of inside.

$A B \cong A B$ by common notion congruencereflexive.

$B$ is on circle $J$ by definition of on.

$D$ is on circle $J$ by definition of on.

$A=A$ by common notion equalityreflexive.

$A$ is inside circle $J$ by definition of inside.

Let $C$ be such that $C$ is on circle $K \wedge C$ is on circle $J$ by postulate circle-circle.

$A C \cong A B$ by axiom circle-center-radius.

$A B \cong A C$ by lemma congruencesymmetric.

$B C \cong B A$ by axiom circle-center-radius.

$B C \cong A B$ by lemma congruenceflip.

$B C \cong A C$ by lemma congruencetransitive.

$A B \cong B C$ by lemma congruencesymmetric.

$A C \cong C A$ by common notion equalityreverse.

$B C \cong C A$ by lemma congruencetransitive.

$A B C$ is equilateral by definition of equilateral.

$B \neq C$ by axiom nocollapse.

$C \neq A$ by axiom nocollapse. 
Let show that $C$ is strictly between $A$ and $B$ does not hold by contradiction:

\{

$A C \nsucceq A B$ by lemma partnotequalwhole.

$C A \cong A C$ by common notion equalityreverse.

$C A \cong A B$ by lemma congruencetransitive.

$A C \cong C A$ by common notion equalityreverse.

$A C \cong A B$ by lemma congruencetransitive.

We have a contradiction.

\}

Let show that $B$ is strictly between $A$ and $C$ does not hold by contradiction:

\{

$A B \nsubseteq A C$ by lemma partnotequalwhole.

$A B \cong C A$ by lemma congruencetransitive.

$C A \cong A C$ by common notion equalityreverse.

$A B \cong A C$ by lemma congruencetransitive.

We have a contradiction.

\}

Let show that $A$ is strictly between $B$ and $C$ does not hold by contradiction:

\{

$B A \nsubseteq B C$ by lemma partnotequalwhole.

$B A \cong A B$ by common notion equalityreverse.

$B A \cong B C$ by lemma congruencetransitive.

We have a contradiction.

\}

Let show that $A B C$ are collinear does not hold by contradiction:

\{

$A \neq C$ by lemma inequalitysymmetric.

$A=B \vee A=C \vee B=C \vee A$ is strictly between $B$ and $C \vee$ $B$ is strictly between $B$ and $C \vee C$ is strictly between $A$ and $B$ by definition of collinear.

We have a contradiction.

\}

$A B C$ is a triangle by definition of triangle.

\section{Appendix B: Axioms and definitions}

The following formulas are presented in a format that can be cut and pasted, even from a pdf file.

\section{Definitions}

$A$ and $B$ are distinct points

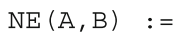

$A, B$, and $C$ are collinear

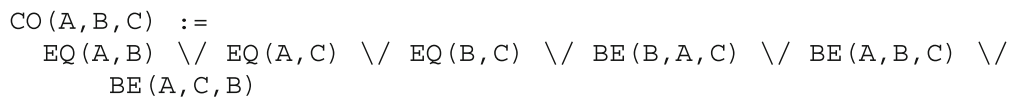


$A, B$, and $C$ are not collinear

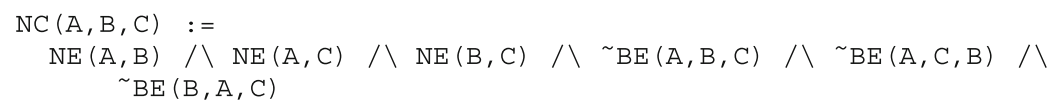

$P$ is inside (some) circle $J$ of center $C$ and radius $A B$

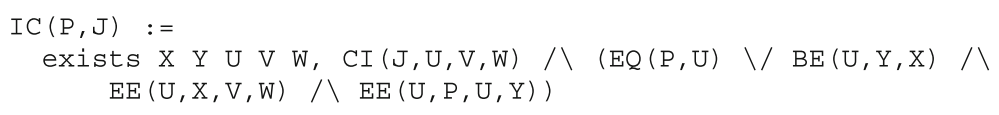

$P$ is outside (some) circle $J$ of center $U$ and radius $V W$

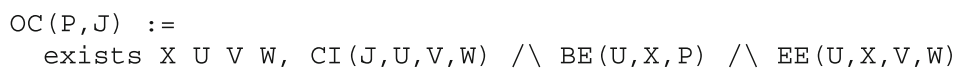

$B$ is on (some) circle $J$ of center $U$ and radius $X Y$

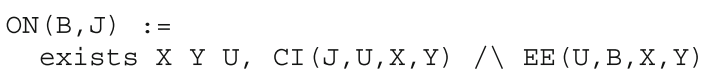

$A B C$ is equilateral

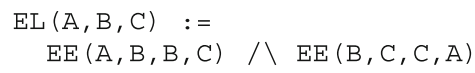

$A B C$ is a triangle

$$
\begin{gathered}
\operatorname{TR}(A, B, C):= \\
\operatorname{NC}(A, B, C)
\end{gathered}
$$

$C$ lies on ray $A B$

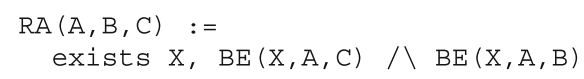

$A B$ is less than $C D$

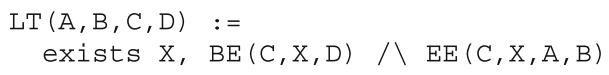

$B$ is the midpoint of $A C$

Angle $A B C$ is equal to angle $a b c$

$$
\begin{aligned}
& \operatorname{MI}(\mathrm{A}, \mathrm{B}, \mathrm{C}):= \\
& \operatorname{BE}(\mathrm{A}, \mathrm{B}, \mathrm{C}) \quad / \backslash \mathrm{EE}(\mathrm{A}, \mathrm{B}, \mathrm{B}, \mathrm{C})
\end{aligned}
$$

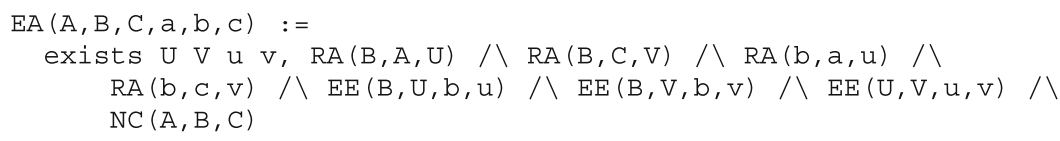


$D B F$ is a supplement of $A B C$

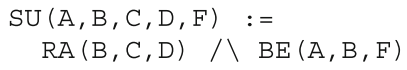

$A B C$ is a right angle

$\operatorname{RR}(A, B, C) \quad:=$ exists $X, B E(A, B, X) / \backslash E E(A, B, X, B) / \triangle E E(A, C, X, C) / N N E(B, C)$

$P Q$ is perpendicular to $A B$ at $C$ and $N C A B P$

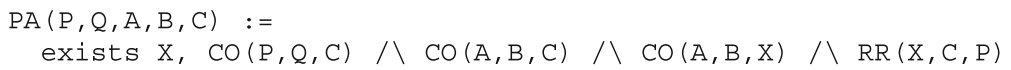

$P Q$ is perpendicular to $A B$

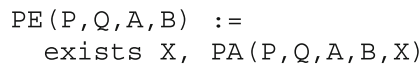

$P$ is in the interior of angle $A B C$

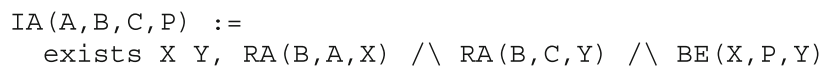

$P$ and $Q$ are on opposite sides of $A B$

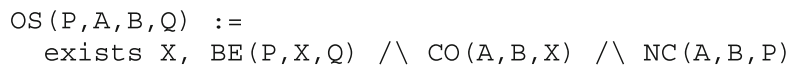

$P$ and $Q$ are on the same side of $A B$

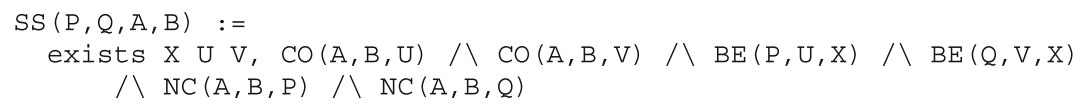

$A B C$ is isosceles with base $B C$

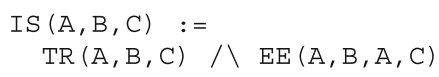

$A B$ cuts $C D$ in $E$

$\mathrm{CU}(\mathrm{A}, \mathrm{B}, \mathrm{C}, \mathrm{D}, \mathrm{E}) \quad:=$

$\mathrm{BE}(\mathrm{A}, \mathrm{E}, \mathrm{B}) / \backslash \mathrm{BE}(\mathrm{C}, \mathrm{E}, \mathrm{D}) / \backslash \mathrm{NC}(\mathrm{A}, \mathrm{B}, \mathrm{C}) / \mathrm{NC}(\mathrm{A}, \mathrm{B}, \mathrm{D})$

Triangle $A B C$ is congruent to abc

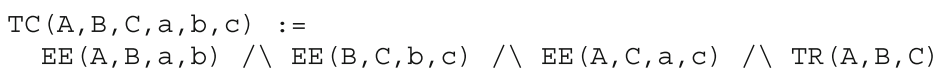


Angle $A B C$ is less than angle DEF

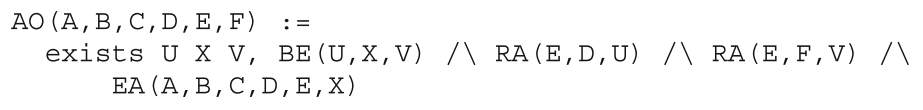

$A B$ and $C D$ are together greater than $E F$

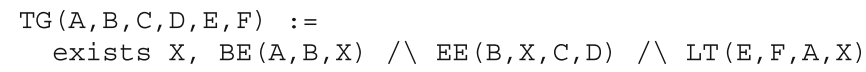

$A B, C D$ are together greater than $E F, G H$

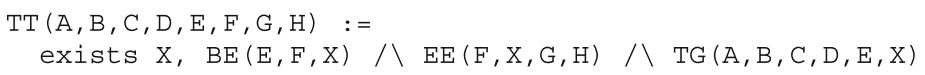

$A B C$ and DEF make together two right angles

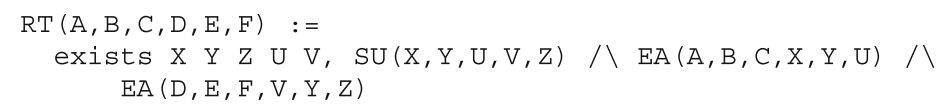

$A B$ meets $C D$

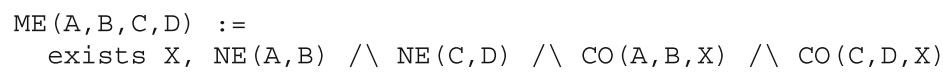

$A B$ crosses $C D$

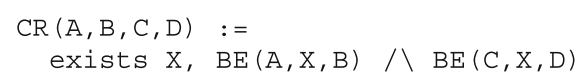

$A B$ and $C D$ are Tarski parallel

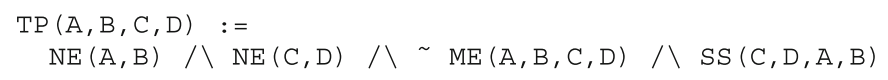

$A B$ and $C D$ are parallel

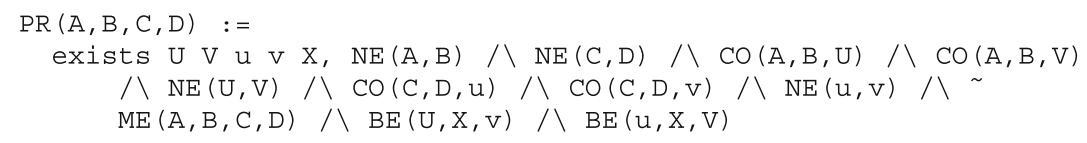

$A B C$ and $D E F$ are together equal to $P Q R$

$\mathrm{AS}(\mathrm{A}, \mathrm{B}, \mathrm{C}, \mathrm{D}, \mathrm{E}, \mathrm{F}, \mathrm{P}, \mathrm{Q}, \mathrm{R}) \quad:=$ exists $X, E A(A, B, C, P, Q, X) / \operatorname{EA}(D, E, F, X, Q, R) / \triangle B E(P, X, R)$

$A B C D$ is a parallelogram

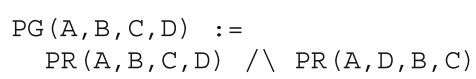


$A B C D$ is a square

$\mathrm{SQ}(\mathrm{A}, \mathrm{B}, \mathrm{C}, \mathrm{D}) \quad:=$

$\mathrm{EE}(\mathrm{A}, \mathrm{B}, \mathrm{C}, \mathrm{D}) / \bigwedge \mathrm{EE}(\mathrm{A}, \mathrm{B}, \mathrm{B}, \mathrm{C}) /(\mathrm{EE}(\mathrm{A}, \mathrm{B}, \mathrm{D}, \mathrm{A}) / \mathrm{RR}(\mathrm{D}, \mathrm{A}, \mathrm{B}) \quad /$ $R R(A, B, C) \wedge R R(B, C, D) \wedge R R(C, D, A)$

$A B C D$ is a rectangle

$\operatorname{RE}(A, B, C, D) \quad:=$

$\mathrm{RR}(\mathrm{D}, \mathrm{A}, \mathrm{B}) / \mathrm{RR}(\mathrm{A}, \mathrm{B}, \mathrm{C}) \wedge \mathrm{RR}(\mathrm{B}, \mathrm{C}, \mathrm{D}) / \mathrm{RR}(\mathrm{C}, \mathrm{D}, \mathrm{A}) / \mathrm{CR}(\mathrm{A}, \mathrm{C}, \mathrm{B}, \mathrm{D})$

$A B C D$ and abcd are congruent rectangles

$R C(A, B, C, D, a, b, C, d):=$ $\operatorname{RE}(\mathrm{A}, \mathrm{B}, \mathrm{C}, \mathrm{D}) / 入 \operatorname{RE}(\mathrm{a}, \mathrm{b}, \mathrm{C}, \mathrm{d}) / 入 \mathrm{EE}(\mathrm{A}, \mathrm{B}, \mathrm{a}, \mathrm{b}) / 入 \mathrm{EE}(\mathrm{B}, \mathrm{C}, \mathrm{b}, \mathrm{c})$

\section{$A B C D$ and abcd are equal rectangles}

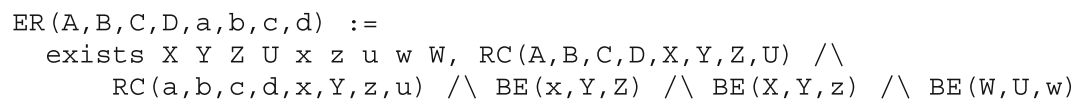

$A B C D$ is a base rectangle of triangle $B C E$

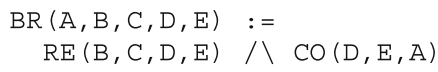

$A B C$ and abc are equal triangles

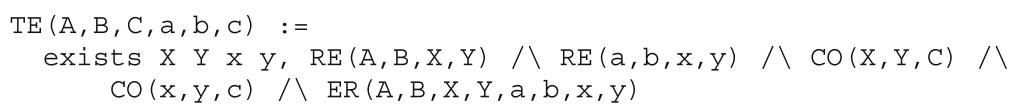

\section{$A B C D$ and abcd are equal quadrilaterals}

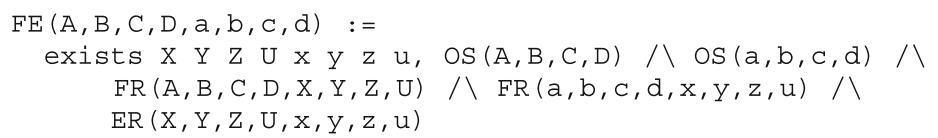

\section{Common notions}

cn-equalitytransitive

forall A B C, EQ $(\mathrm{A}, \mathrm{C}) / 入 \mathrm{EQ}(\mathrm{B}, \mathrm{C})==>\mathrm{EQ}(\mathrm{A}, \mathrm{B})$

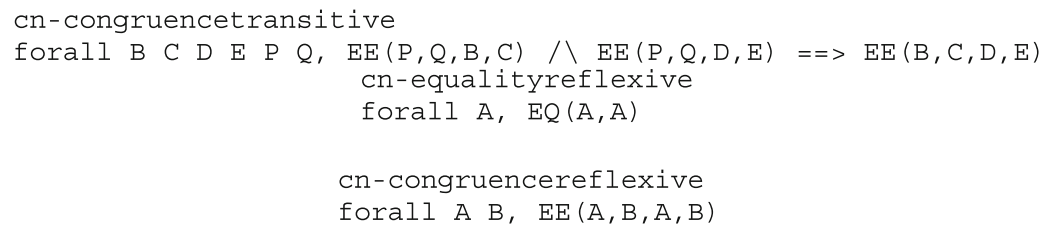


cn-equalityreverse

forall $A B, E E(A, B, B, A)$

cn-sumofparts

forall A B C a b C, $\mathrm{EE}(\mathrm{A}, \mathrm{B}, \mathrm{a}, \mathrm{b}) / \backslash \mathrm{EE}(\mathrm{B}, \mathrm{C}, \mathrm{b}, \mathrm{C}) / \mathrm{BE}(\mathrm{A}, \mathrm{B}, \mathrm{C}) /$ $\operatorname{BE}(\mathrm{a}, \mathrm{b}, \mathrm{c})==>\operatorname{EE}(\mathrm{A}, \mathrm{C}, \mathrm{a}, \mathrm{C})$

cn-stability

forall $\mathrm{A} \mathrm{B}, \sim \mathrm{NE}(\mathrm{A}, \mathrm{B})==\mathrm{EQ}(\mathrm{A}, \mathrm{B})$

cn-equalitysub

forall A B C D, $\mathrm{EQ}(\mathrm{D}, \mathrm{A}) / \mathrm{BE}(\mathrm{A}, \mathrm{B}, \mathrm{C})==\mathrm{BE}(\mathrm{D}, \mathrm{B}, \mathrm{C})$

\section{Axioms of betweenness and congruence}

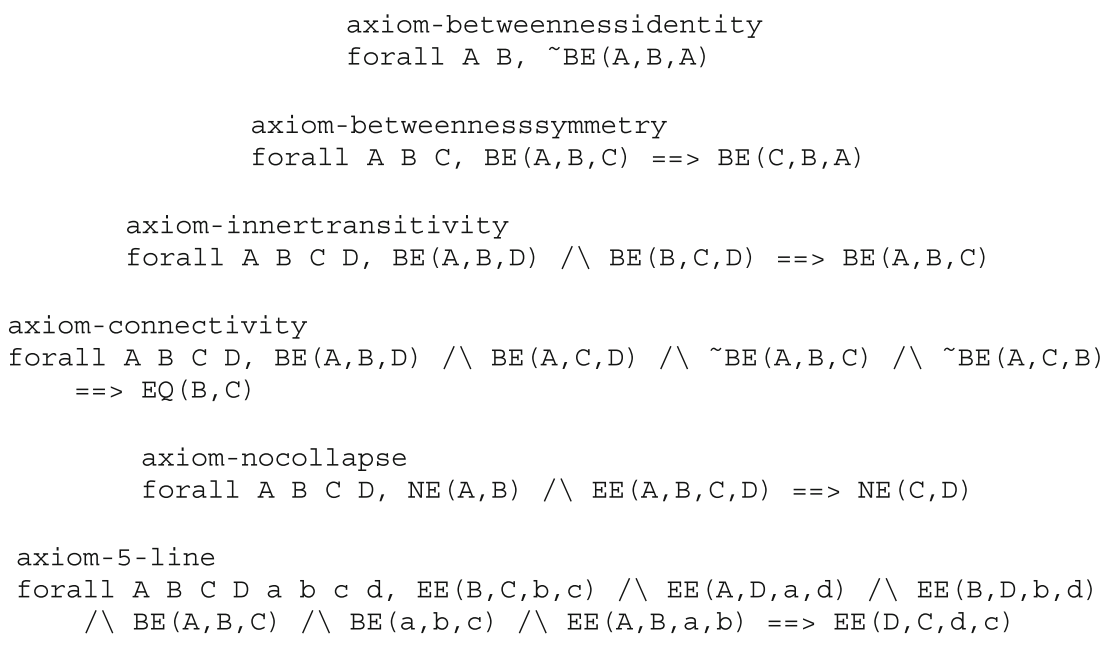

\section{Postulates}

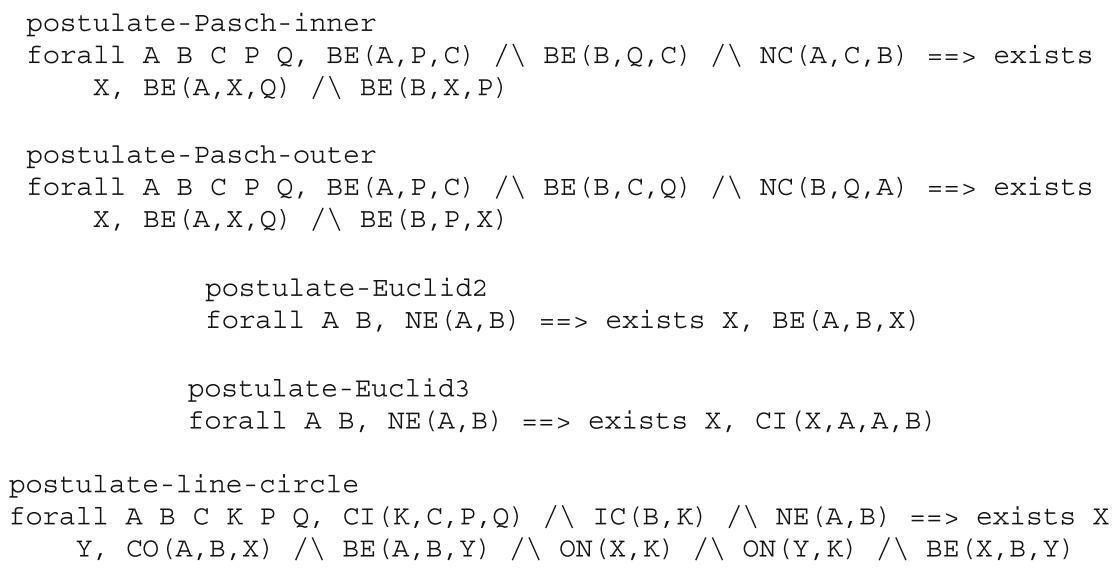




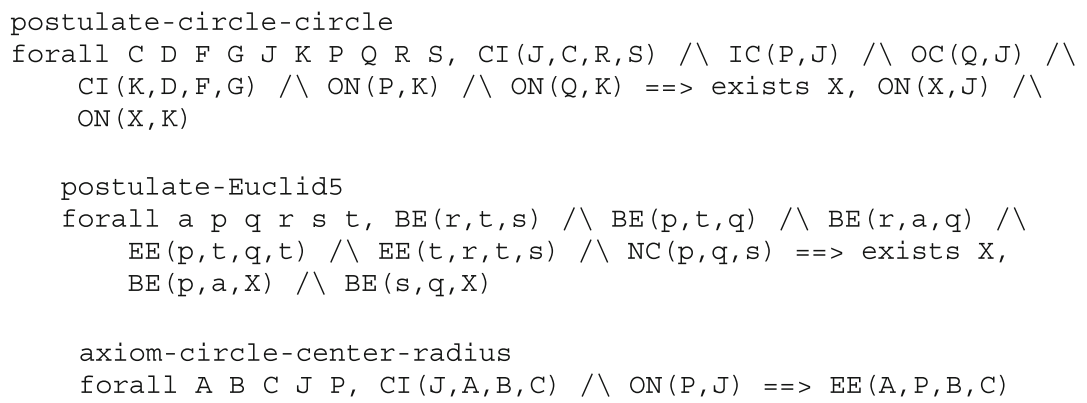

\section{Axioms for equal figures}

axiom-congruentequal

forall A B C a b C, TC $(A, B, C, a, b, C)==\operatorname{ET}(A, B, C, a, b, C)$

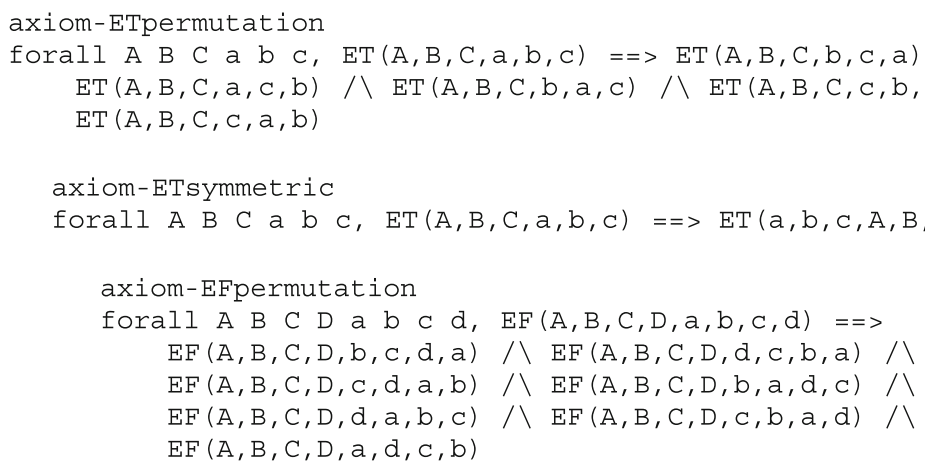

axiom-halvesofequals

forall A B C D a b c d, $\operatorname{ET}(A, B, C, B, C, D) / \mathrm{OS}(A, B, C, D) \quad 八$ $\operatorname{ET}(a, b, c, b, c, d) \quad \bigwedge O S(a, b, c, d) \quad / E F(A, B, D, C, a, b, d, c)==>$ $\operatorname{ET}(A, B, C, a, b, c)$

axiom-EFsymmetric

forall A B C D a b $\mathrm{C} d, \operatorname{EF}(A, B, C, D, a, b, c, d)==\operatorname{EF}(a, b, c, d, A, B, C, D)$

axiom-EFtransitive

forall A B C D P Q R S a b c d, EF(A, B, C, D, a,b,c,d) 八 $E F(a, b, c, d, P, Q, R, S)==\operatorname{EF}(A, B, C, D, P, Q, R, S)$

axiom-ETtransitive

forall A B C P Q R a b c, $\operatorname{ET}(A, B, C, a, b, c) / N E T(a, b, c, P, Q, R)==>$ $\operatorname{ET}(A, B, C, P, Q, R)$

axiom-cutoff 1

forall A B C D E a b c d e, $\operatorname{BE}(\mathrm{A}, \mathrm{B}, \mathrm{C}) / \mathrm{BE}(\mathrm{a}, \mathrm{b}, \mathrm{c}) / \mathrm{BE}(\mathrm{E}, \mathrm{D}, \mathrm{C}) /$ $\mathrm{BE}(e, d, c) /(\operatorname{ET}(B, C, D, b, c, d) / \operatorname{ET}(A, C, E, a, c, e)==>$ $\mathrm{EF}(\mathrm{A}, \mathrm{B}, \mathrm{D}, \mathrm{E}, \mathrm{a}, \mathrm{b}, \mathrm{d}, \mathrm{e})$

axiom-cutoff2

forall A B C D E a b c d e, $\operatorname{BE}(B, C, D) /(B E(b, c, d) /$ $E T(C, D, E, C, d, e) / \triangle E F(A, B, D, E, a, b, d, e)==\operatorname{EF}(A, B, C, E, a, b, c, e)$ 


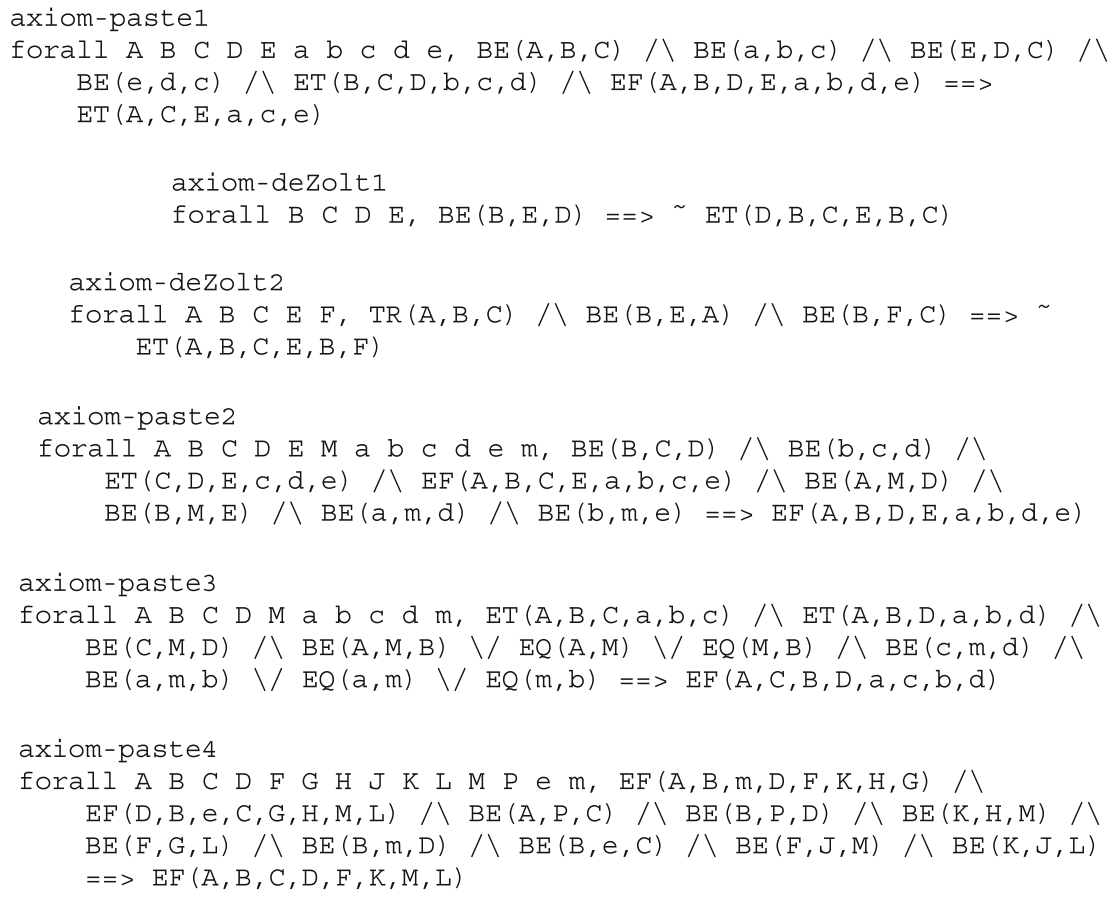

Publisher's note Springer Nature remains neutral with regard to jurisdictional claims in published maps and institutional affiliations.

\section{References}

1. Avigad, J., Dean, E., Mumma, J.: A formal system for Euclid's Elements. Rev. Symb. Log. 2, 700-768 (2009)

2. Beeson, M.: A constructive version of Tarski’s geometry. Ann. Pure Appl. Logic 166, 1199-1273 (2015)

3. Beeson, M.: Constructive geometry and the parallel postulate. Bull. Symb. Log. 22, 1-104 (2016)

4. Bledsoe, W.W., Loveland, D.: Automated Theorem Proving: After 25 Years. American Mathematical Society, Providence (1983)

5. Boutry, P.: On the Formalization of Foundations of Geometry. PhD thesis, University of Strasbourg (2018)

6. Boutry, P., Braun, G., Narboux, J.: From Tarski to Descartes: formalization of the arithmetization of Euclidean geometry. In: Davenport, J.H., Ghourabi, F. (eds.) SCSS 2016, the 7th International Symposium on Symbolic Computation in Software Science, vol. 39 of EPiC Series in Computing, Tokyo, Japan, EasyChair, pp. 14-28 (2016)

7. Boutry, P., Braun, G., Narboux, J.: Formalization of the arithmetization of Euclidean plane geometry and applications. J. Symb. Comput. 90, 149-168 (2019). https://doi.org/10.1016/j.jsc.2018.04.007

8. Boutry, P., Gries, C., Narboux, J., Schreck, P.: Parallel postulates and continuity axioms: a mechanized study in intuitionistic logic using Coq. J. Autom. Reason., p. 68. https://link.springer.com/article/10.1007 \%2Fs10817-017-9422-8 (2017)

9. Braun, G., Boutry, P., Narboux, J.: From Hilbert to Tarski. In: 11th International Workshop on Automated Deduction in Geometry, Proceedings of ADG 2016, Strasbourg, France, p. 19 (2016)

10. Braun, G., Narboux, J.: From Tarski to Hilbert. In: Ida, T., Fleuriot, J. (eds.) Automated Deduction in Geometry 2012 vol. 7993, Edinburgh, United Kingdom, Jacques Fleuriot, Springer, pp. 89-109 (2012)

11. Braun, G., Narboux, J.: A synthetic proof of Pappus' theorem in Tarski's geometry. J. Autom. Reason. 58, 23 (2017)

12. Chou, S.C.: Mechanical Geometry Theorem Proving. Kluwer Academic Publishers, Norwell (1987) 
13. Chou, S.C.: An introduction to Wu's method for mechanical theorem proving in geometry. J. Autom. Reason. 4, 237-267 (1988)

14. Chou, S.-C., Gao, X.-S., Zhang, J.-Z.: Machine Proofs in Geometry: Automated Production of Readable Proofs for Geometry Theorems. World Scientific (1994)

15. Chou, S.C., Schelter, W.F.: Proving geometry theorems with rewrite rules. J. Autom. Reason. 2, 253-273 (1986)

16. Corbineau, P.: A declarative language for the Coq proof assistant. In: Types for Proofs and Programs, vol. 4941 of LNCS, Springer (2008)

17. Euclid: The Elements of Euclid, viz. the first six books, together with the eleventh and the twelfth, Nourse and Balfous, Edinburgh, 7th ed., 1787. Translated by Robert Simson. Available from Bibliotheque Nationale at http://gallica.bnf.fr/ark:/12148/bpt6k1163221v

18. Euclid: The Thirteen Books of the Elements. Dover, New York (1956). Three volumes. Includes commentary by the translator, Sir Thomas L. Heath

19. Gelernter, H.: Realization of a geometry theorem-proving machine. In: Feigenbaum, E., Feldman, J. (eds.) Computers and Thought, pp. 134-152. McGraw-Hill, New York (1963)

20. Gelernter, H., Hansen, J.R., Loveland, D.W.: Empirical explorations of a geometry-theorem proving machine. In: Feigenbaum, E., Feldman, J. (eds.) Computers and Thought, pp. 153-167. McGraw-Hill, New York (1963)

21. Greenberg, M.J. Euclidean and non-Euclidean Geometries: Development and History, 4th edn. W. H. Freeman, New York (2008)

22. Gries, C.: Axiomes de continuité en géométrie neutre : Une étude méchanisée en Coq. University of Strasbourg, internship report (2017)

23. Gupta, H.N.: Contributions to the Axiomatic Foundations of Geometry. PhD Thesis, University of California, Berkeley (1965)

24. Hartshorne, R.: Geometry: Euclid and Beyond. Springer, New York (2000)

25. Hilbert, D.: Foundations of Geometry (Grundlagen der Geometrie). Open Court, La Salle, Illinois Second English edition, translated from the tenth German edition by Leo Unger. Original publication year (1899)

26. Kapur, D.: A refutational approach to geometry theorem proving. J. Artif. Intell. 37, 61-93 (1988)

27. Kutzler, B., Stifter, S.: Automated geometry theorem proving using Buchberger's algorithm. In: Proceedings of the International Symposium on Symbolic and Algebraic Computation (SYMSAC 86), Waterloo, 1986, B. Char, ed., pp. 209-214 (1986)

28. Manders, K.: The Euclidean diagram (1995). In: The Philosophy of Mathematical Practice, Paolo Mancosu. Oxford University Press ed. (2011)

29. Miller, N.: A Diagrammatic Formal System for Euclidean Geometry. PhD thesis, Cornell University (2001)

30. Miller, N.: Euclid and his twentieth century rivals: diagrams in the logic of Euclidean geometry. Studies in the theory and applications of diagrams, CSLI Publications, Stanford, Calif, 2007. OCLC: ocm71947628

31. Miller, N.: On the inconsistency of Mumma's Eu. Notre Dame Journal of Formal Logic 53, 27-52 (2012)

32. Mollerup, J.: Die Beweise der ebenen Geometrie ohne Benutzung der Gleichheit und Ungleichheit der Winkel. Math. Ann. 58, 479-496 (1904)

33. Mumma, J.: Proofs, pictures, and Euclid. Synthese 175, 255-287 (2010)

34. Narboux, J., Janičić, P., Fleuriot, J.: Computer-assisted theorem proving in synthetic geometry. In: Sitharam, M., John, A.S., Sidman, J. (eds.) Handbook of Geometric Constraint Systems Principles, Chapman and Hall/CRC, ch. 2. in press (2018)

35. Pasch, M.: Vorlesung über Neuere Geometrie. Teubner, Leipzig (1882)

36. Peano, G.: Principii de Geometria. Fratelli Bocca, Torino (1889)

37. Potts, R.: Euclid's Elements of Geometry [Books 1-6, 11,12] with Explanatory Notes; Together with a Selection of Geometrical Exercises. To which is Prefixed an Intr., Containing a Brief Outline of the History of Geometry. Oxford University Press, Oxford (1845)

38. Proclus: A Commentary on the First Book of Euclid. Princeton University Press, Princeton (1970)

39. Quaife, A.: Automated development of Tarski's geometry. J. Autom. Reason. 5, 97-118 (1989)

40. Schwabhäuser, W., Szmielew, W., Tarski, A.: Metamathematische Methoden in der Geometrie: Teil I: Ein axiomatischer Aufbau der euklidischen Geometrie. Teil II: Metamathematische Betrachtungen (Hochschultext). Springer-Verlag, 1983. Reprinted 2011 by Ishi Press, with a new foreword by Michael Beeson

41. Stojanovic Durdevic, S., Narboux, J., Janicic, P.: Automated generation of machine verifiable and readable proofs: a case study of Tarski’s geometry. Ann. Math. Artif. Intell. 73, 25 (2015) 
42. Strommer, J.: Über die Kreisaxiome. Period. Math. Hung. 4, 3-16 (1973)

43. Tarski, A., Givant, S.: Tarski's system of geometry. Bull. Symb. Log. 5, 175-214 (1999)

44. Veronese, G.: Fondamenti di geometria a più dimensioni e a più specie di unità rettilinee esposti in forma elementare. Lezioni per la scuola di magistero in matematica, Padova: Tipografia del Seminario, 1891 (1891)

45. Wu, W.-T.: On the decision problem and the mechanization of theorem-proving in elementary geometry. Sci. Sinica 21, reprinted in [4] (1978)

46. Wu, W.-T.: Mechanical Theorem Proving in Geometries: Basic Principles. Springer, Wien/ New York (1994) 